\title{
Testing grain surface chemistry: a survey of deuterated formaldehyde and methanol in low-mass class 0 protostars ${ }^{\star} \star \star \star$
}

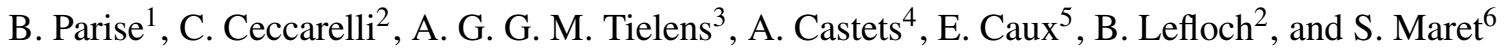 \\ 1 Max Planck Institut für Radioastronomie, Auf dem Hügel 69, 53121 Bonn, Germany \\ 2 Laboratoire d'Astrophysique, Observatoire de Grenoble, BP 53, 38041 Grenoble Cedex 09, France \\ 3 SRON, PO Box 800, 9700 AV Groningen, The Netherlands \\ 4 Observatoire de Bordeaux, BP 89, 33270 Floirac, France \\ 5 CESR CNRS-UPS, BP 4346, 31028 Toulouse Cedex 04, France \\ ${ }^{6}$ Department of Astronomy, University of Michigan, 500 Church Street, Ann Arbor MI 48109-1042, USA
}

Received 4 November 2005 / Accepted 28 February 2006

ABSTRACT

\begin{abstract}
Context. Despite the low cosmic abundance of deuterium $\left(\mathrm{D} / \mathrm{H} \sim 10^{-5}\right)$, high degrees of deuterium fractionation in molecules are observed in star-forming regions with enhancements that can reach 13 orders of magnitude, a level that current models have difficulty accounting for.

Aims. Multi-isotopologue observations are a very powerful constraint for chemical models. The aim of our observations is to understand the processes that form the observed high abundances of methanol and formaldehyde in low-mass protostellar envelopes (gas-phase processes? chemistry on the grain surfaces?), as well as to better constrain the chemical models.

Methods. With the IRAM $30 \mathrm{~m}$ single-dish telescope, we observed deuterated formaldehyde ( $\mathrm{HDCO}$ and $\left.\mathrm{D}_{2} \mathrm{CO}\right)$ and methanol $\left(\mathrm{CH}_{2} \mathrm{DOH}, \mathrm{CH}_{3} \mathrm{OD}\right.$, and $\left.\mathrm{CHD}_{2} \mathrm{OH}\right)$ towards a sample of seven low-mass class 0 protostars. Using population diagrams, we then derived the fractionation ratios of these species (abundance ratio between the deuterated molecule and its main isotopologue) and compared them to the predictions of grain chemistry models.

Results. These protostars show a similar level of deuteration as in IRAS 16293-2422, where doubly-deuterated methanol - and even triply-deuterated methanol - were first detected. Our observations point to the formation of methanol on the grain surfaces, while formaldehyde formation cannot be fully pinned down. While none of the scenarii can be excluded (gas-phase or grain chemistry formation), they both seem to require abstraction reactions to reproduce the observed fractionations.
\end{abstract}

Key words. ISM: abundances - ISM: molecules - stars: formation

\section{Introduction}

In the past few years, observations of low-mass protostars have revealed unexpected high abundances of deuterated molecules, particularly doubly-deuterated molecules. The discovery of an extremely large amount $\left(\mathrm{D}_{2} \mathrm{CO} / \mathrm{H}_{2} \mathrm{CO} \sim 10 \%\right)$ of doubly-deuterated formaldehyde in the low-mass protostar IRAS 16293-2422 (hereafter IRAS 16293; Ceccarelli et al. 1998) was followed by observations of this same molecule towards a large sample of low-mass protostars (Loinard et al. 2002). The fractionation ratios appeared to be similarly large towards all targeted low-mass protostars.

The suggested interpretation was that such large deuteration is obtained during the cold and dense precollapse phase of the low-mass protostars: highly-deuterated ices are very likely formed via active grain surface chemistry (Tielens 1983), then stored in the grain mantles, and eventually released in the gas phase during the collapse, when the heating of the newly-formed protostar evaporates the ices (Ceccarelli et al. 2001). Strong support for this scheme comes both from the high abundance of $\mathrm{D}_{2} \mathrm{CO}$ observed in prestellar cores (Bacmann et al. 2003) and

* Based on observations with the IRAM $30 \mathrm{~m}$ telescope at Pico Veleta (Spain). IRAM is funded by the INSU/CNRS (France), the MPG (Germany) and the IGN (Spain).

$\star \star$ Figures 7-12 and Tables 6-9 are only available in electronic form at http://www.edpsciences.org from the discovery of the large fractionation of $\mathrm{H}_{3}^{+}$in the same objects (Caselli et al. 2003; Vastel et al. 2004). While these last observations undoubtedly supported the idea that deuteration sets in just before the collapse, the leading chemical process was still largely unproven. In this context, methanol is a key molecule. Indeed, it is believed to be a grain surface product as gas-phase processes are not efficient enough to account for the high abundances observed in star-forming regions (Herbst 2005). Methanol may be the last step in CO hydrogenation on the grain surfaces after the formaldehyde formation. If formaldehyde formation is also dominated by grain chemistry, the deuteration of both molecules is expected to be tightly linked.

Three years ago, doubly-deuterated methanol was detected towards the low-mass protostar IRAS 16293 $\left(\mathrm{CHD}_{2} \mathrm{OH} / \mathrm{CH}_{3} \mathrm{OH} \sim 20 \%\right.$, Parise et al. 2002), basically confirming the grain chemistry scheme. However, the $\mathrm{CH}_{2} \mathrm{DOH} / \mathrm{CH}_{3} \mathrm{OD}$ ratio was found to be unexpectedly large ( $20 \pm 14$, Parise et al. 2002) compared to the value of 3 predicted by the models (Charnley et al. 1997). The proposed hypothesis of a rapid conversion of $\mathrm{CH}_{3} \mathrm{OD}$ into $\mathrm{CH}_{3} \mathrm{OH}$ in the gas phase due to protonation reactions that would affect only the species for which the deuterium is bound to the very electronegative oxygen (Charnley et al. 1997) was also suggested by the model of Osamura et al. (2004). The high observed fractionation of $\mathrm{CH}_{2} \mathrm{DOH}$ and $\mathrm{CHD}_{2} \mathrm{OH}$ was consistent with formation of methanol on the grain surfaces, but required an atomic $\mathrm{D} / \mathrm{H}$ ratio 
Table 1. Parameters of the sources targeted in our deuterated formaldehyde and methanol observations. IRAS 16293-2422 is added for comparison. The offsets used for the OFF position of the position switching mode are indicated in the last column.

\begin{tabular}{ccccccccccc}
\hline \hline Source & $\alpha(2000)$ & $\delta(2000)$ & Region & $\begin{array}{c}\text { Distance } \\
(\text { parsec })\end{array}$ & $\begin{array}{c}L_{\text {bol }}{ }^{a} \\
\left(L_{\odot}\right)\end{array}$ & $\begin{array}{c}M_{\text {env }}{ }^{b} \\
\left(M_{\odot}\right)\end{array}$ & $\begin{array}{c}L_{\text {smm }} / L_{\text {bol }}{ }^{a} \\
(\%)\end{array}$ & $\begin{array}{c}T_{\text {bol }} \\
(\mathrm{K})\end{array}$ & $\begin{array}{c}V_{\text {LSR }} \\
\left(\mathrm{km} \mathrm{s}^{-1}\right)\end{array}$ & $\begin{array}{c}\text { offsets } \\
(\operatorname{arcsec})\end{array}$ \\
\hline IRAS4A & $03: 29: 10.3$ & $31: 13: 32$ & Perseus & 220 & 6 & 2.3 & 5 & 34 & +7 & $(-150,240)$ \\
IRAS4B & $03: 29: 12.0$ & $31: 13: 09$ & Perseus & 220 & 6 & 2.0 & 3 & 36 & +7 & $(-180,260)$ \\
IRAS2 & $03: 28: 55.4$ & $31: 14: 35$ & Perseus & 220 & 16 & 1.7 & $\leq 1$ & 50 & +7 & $(70,180)$ \\
L1448N & $03: 25: 36.3$ & $30: 45: 15$ & Perseus & 220 & 6 & 3.5 & 3 & 55 & +5 & $(300,300)$ \\
L1448mm & $03: 25: 38.8$ & $30: 44: 05$ & Perseus & 220 & 5 & 0.9 & 2 & 60 & +5 & $(300,300)$ \\
L1157mm & $20: 39: 06.2$ & $68: 02: 22$ & Isolated & 325 & 11 & 1.6 & 5 & 60 & +5 & $(150,0)$ \\
L1527 & $04: 39: 53.9$ & $26: 03: 10$ & Taurus & 140 & 2 & 0.9 & 0.7 & 60 & +5 & $(240,0)$ \\
\hline IRAS 16293 & $16: 32: 22.6$ & $-24: 28: 33.0$ & $\rho$-Ophiucus & 160 & 27 & 5.4 & 2 & 43 & +4 & \\
\hline
\end{tabular}

${ }^{a}$ From André et al. (2000) and Cernis (1990).

${ }^{b}$ From Jørgensen et al. (2002).

in the gas-phase as high as $0.1-0.2$ during the mantle formation. This value was challenging gas-phase models at that time, and led Parise et al. (2002) to suggest that "a key parameter was missing" in the chemical schemes. Indeed, this key parameter was soon discovered. In molecular clouds, the main reservoir of deuterium is molecular HD. Some deuterium can be transferred from this reservoir to other molecules by the intermediate of the ion $\mathrm{H}_{2} \mathrm{D}^{+}$that forms according to the exothermic reaction: $\mathrm{HD}+\mathrm{H}_{3}^{+} \rightarrow \mathrm{H}_{2}+\mathrm{H}_{2} \mathrm{D}^{+}$. Collision of $\mathrm{CO}$ with $\mathrm{H}_{2} \mathrm{D}^{+}$, followed by recombination, will then form atomic deuterium. Depletion of $\mathrm{CO}-$ one of the main destruction agents of $\mathrm{H}_{2} \mathrm{D}^{+}-$drives the fractionation of this key intermediary in the gas-phase deuterium fractionation schemes all the way to $\mathrm{HD}_{2}^{+}$and even $\mathrm{D}_{3}^{+}$. The inclusion in the gas-phase schemes of multiply-deuterated isotopomers of $\mathrm{H}_{2} \mathrm{D}^{+}$as new intermediate molecules for deuterium transfer from the HD main reservoir allowed prediction of the atomic $\mathrm{D} / \mathrm{H}$ ratio required by methanol observations (Roberts et al. 2003). Triply-deuterated methanol was later detected in IRAS 16293 (Parise et al. 2004), and the $\mathrm{CH}_{3} \mathrm{OH}$ column density was better evaluated by analyzing ${ }^{13} \mathrm{CH}_{3} \mathrm{OH}$ transitions. The observed $\mathrm{CD}_{3} \mathrm{OH} / \mathrm{CH}_{3} \mathrm{OH}$ fractionation ratio, which was found to be consistent with the $\mathrm{CH}_{2} \mathrm{DOH}$ and $\mathrm{CHD}_{2} \mathrm{OH}$ fractionations, allowed the grain chemistry scheme to be confirmed. While much progress has been made through laboratory (e.g. Nagaoka et al. 2005) and theoretical studies to understand the observed high deuterium fractionation in IRAS 16293, observations of a larger sample are required to determine whether this is a common phenomena in protostellar environments.

In this paper, we report observations of singly (HDCO) and doubly $\left(\mathrm{D}_{2} \mathrm{CO}\right)$ deuterated formaldehyde and singly-deuterated methanol $\mathrm{CH}_{2} \mathrm{DOH}$ and $\mathrm{CH}_{3} \mathrm{OD}$, as well as doubly-deuterated $\mathrm{CHD}_{2} \mathrm{OH}$, towards a sample of low-mass protostars. This article is organized as follows: observations are presented in Sect. 2, analysis of the data is presented in Sect. 3, the results are discussed in Sect. 4, and conclusions drawn in Sect. 5.

\section{Observations and results}

\subsection{Observations}

Using the IRAM 30-m telescope (Pico Veleta, Spain), we observed the five deuterated species $\mathrm{HDCO}, \mathrm{D}_{2} \mathrm{CO}, \mathrm{CH}_{2} \mathrm{DOH}$, $\mathrm{CH}_{3} \mathrm{OD}$, and $\mathrm{CHD}_{2} \mathrm{OH}$ towards the six low-mass protostars NGC1333-IRAS4A, -IRAS4B, -IRAS2, L1448N, L1448mm, and $\mathrm{L} 1157 \mathrm{~mm}$. We also present observations of deuterated formaldehyde (HDCO and $\mathrm{D}_{2} \mathrm{CO}$ ) towards L1527. All these sources, already studied by Maret et al. (2004), are
Class 0 protostars, i.e. in the early phase of the gravitational collapse. Table 1 summarizes the main characteristics of these sources.

The observations were performed in September and November 2002, September 2003, and March 2004. For methanol, four receivers were used simultaneously at 3, 3, 1.3, and $1.3 \mathrm{~mm}$ with typical system temperatures of about 100 , 150,400 , and $400 \mathrm{~K}$, respectively. These receivers were connected to the VESPA autocorrelator, divided into 6 units. For formaldehyde, four receivers were used simultaneously at 3,2, 1.3 , and $1 \mathrm{~mm}$, with typical system temperatures of about 110 , 220,300 , and $400 \mathrm{~K}$, connected to VESPA. The telescope beam width varies between $30^{\prime \prime}$ at $83 \mathrm{GHz}$ and $9^{\prime \prime}$ at $276 \mathrm{GHz}$. All observations were performed using the position switching mode. The offset positions are summarized in Table 1 . The pointing accuracy was monitored regularly on strong extragalactic continuum sources and found to be better than $3^{\prime \prime}$. Our spectra for deuterated methanol were obtained with integration times ranging from 280 to 420 min depending on the source.

All fluxes are indicated in units of main-beam temperature, and the error bars were calculated as the quadratic sum of the statistical noise (noise rms of the data) and the calibration uncertainty. In order to account for the atmospheric calibration, as well as for uncertainties in the band rejection, and taking into account that most of our observations were performed at high elevation, we adopted the following calibration uncertainties: $5 \%$ for lines observed at frequencies lower than $130 \mathrm{GHz}, 10 \%$ for frequencies between 130 and $260 \mathrm{GHz}$, and $15 \%$ for higher frequencies.

All upper limits are given at a $3 \sigma$ level:

$\int T_{\mathrm{mb}} \mathrm{d} v \leq 3 \sigma(1+\alpha) \sqrt{\delta v \cdot \Delta v}$

where $\alpha$ is the calibration uncertainty indicated above, $\sigma$ the noise rms of the observations, $\delta \mathrm{v}$ the spectral resolution, and $\Delta \mathrm{v}$ the assumed linewidth $\left(1.5 \mathrm{~km} \mathrm{~s}^{-1}\right.$ for $\mathrm{D}_{2} \mathrm{CO}, 3 \mathrm{~km} \mathrm{~s}^{-1}$ for deuterated methanol), based on observations of detected lines.

The observed fluxes are listed in Tables 6-9. Examples of observed spectra are shown in Fig. 7-12.

\subsection{Results}

We detected the two deuterated formaldehyde isotopes (HDCO and $\mathrm{D}_{2} \mathrm{CO}$ ) towards all the sources in our sample. Regarding methanol, only the three sources NGC 1333-IRAS4A, -IRAS4B, and -IRAS2 have adequate detections. This is consistent with the study by Maret et al. (2005) that shows that these three sources are indeed the 
brightest ones for $\mathrm{CH}_{3} \mathrm{OH}$ emission. $\mathrm{CH}_{2} \mathrm{DOH}$ was detected in all sources where it was searched for, but only the low-lying transition was detected in the case of L1448N, L1448mm, and $\mathrm{L} 1157 \mathrm{~mm}$. $\mathrm{CH}_{3} \mathrm{OD}$ was detected only towards IRAS4A, IRAS4B, L1448mm, and L1157mm, with only one transition detected in the two last sources. Finally, doubly-deuterated methanol was detected towards IRAS4A, IRAS4B, and IRAS2. Upper limits were derived for the other sources. Despite the substantial integration time (420 min for L1448N, $108 \mathrm{~min}$ for L1448mm, and $264 \mathrm{~min}$ for L1157mm), upper limits on the fractionation are not very significant, mostly because of the low $\mathrm{CH}_{3} \mathrm{OH}$ abundance in those sources (Maret et al. 2005).

The observed lines are relatively narrow, around 1 to $1.5 \mathrm{~km} \mathrm{~s}^{-1}$ for deuterated formaldehyde and up to $3 \mathrm{~km} \mathrm{~s}^{-1}$ for deuterated methanol. For formaldehyde, in particular, the linewidths for the deuterated species are smaller than for the main isotopomer (Maret et al. 2004). Indeed, the low energy $\mathrm{H}_{2} \mathrm{CO}$ lines are probably contaminated by an outflow contribution, as discussed in Maret et al. (2004). The narrow lines emitted by the deuterated isotopomers of $\mathrm{H}_{2} \mathrm{CO}$ suggest that the emission is dominated in this case by the cold outer envelope of the protostar, as expected from those lines with relatively low upper energies. This is consistent with the observations of extended emission of $\mathrm{D}_{2} \mathrm{CO}$ in the low-mass protostar IRAS 16293-2422 (Ceccarelli et al. 2001). This observation was interpreted as an indication that $\mathrm{D}_{2} \mathrm{CO}$ comes from the evaporation of CO-rich ices that evaporate around $20 \mathrm{~K}$, i.e. at a lower temperature than polar ices. For methanol, the signal-to-noise ratio is too low to draw any firm conclusion, but the deuterated lines also seem to be narrower than the main isotopomer, suggesting that the emission in this case is also dominated by the envelope contribution. However, the larger linewidths compared to formaldehyde, as well as the higher rotational temperature (see next paragraph), suggest that it probes the envelope more deeply.

\section{Analysis}

The analysis based on an accurate model of infalling envelope of $\mathrm{H}_{2} \mathrm{CO}$ and $\mathrm{CH}_{3} \mathrm{OH}$ transitions towards the sample of lowmass protostars showed that the abundance of those two species jumps in the inner warm part of the envelope (Maret et al. 2004, 2005). This abundance jump has been attributed to the evaporation of $\mathrm{H}_{2} \mathrm{CO}$ and $\mathrm{CH}_{3} \mathrm{OH}$ from polar ices in the region where the temperature is higher than $100 \mathrm{~K}$. In principle, a multifrequency analysis could be done for the $\mathrm{CH}_{2} \mathrm{DOH}$ molecule, for several lines have been observed in this molecule. However, the analysis could only be done in the LTE approximation, for the collisional coefficients are not known. Since we cannot perform the analysis for the other molecules, we would not be able to make any comparison anyway. We therefore decided not to implement this analysis in this article. We will instead use the rotational diagram technique, which gives the column density averaged on the source extent for optically thin and LTE lines. Both conditions are likely to be correct in our case, first because we do not expect particularly large column densities and second because the critical densities ${ }^{1}$ for these lines are around $10^{5}$ to $10^{6} \mathrm{~cm}^{-3}$, which are about the densities around these sources (Maret et al. 2004).

${ }^{1}$ For example, for $\mathrm{CH}_{2} \mathrm{DOH}$, the Einstein coefficients for the observed transitions lie in the $10^{-6}$ to $10^{-5} \mathrm{~s}^{-1}$ range. The collisional deexcitation rates are expected to be on the order of $10^{-11} \mathrm{~cm}^{3} \mathrm{~s}^{-1}$, leading to critical densities of $10^{5}$ to $10^{6} \mathrm{~cm}^{3}$.
The lines have been observed at several different frequencies, hence with different spatial resolutions. Indeed, the beam size of the IRAM $30 \mathrm{~m}$ telescope is $30^{\prime \prime}$ at $83 \mathrm{GHz}$ and $9^{\prime \prime}$ at $276 \mathrm{GHz}$. If the source is smaller than the beam size of the observation, the derived column density must be corrected for the beam dilution. Unfortunately, we are not certain of the size of the deuterated formaldehyde and methanol emission in the targetted protostars. In order to check to what extent the source size affects the derived column densities and fractionations, we discuss in the following paragraph a detailed study of the $\mathrm{CH}_{2} \mathrm{DOH}$ data on IRAS4A.

\subsection{Constraints on the size of the $\mathrm{CH}_{2} \mathrm{DOH}$}

We plotted the rotational diagrams of $\mathrm{CH}_{2} \mathrm{DOH}$ towards IRAS4A (the source with the largest number of detected lines) assuming different sizes for the source emission: $10^{\prime \prime}, 15^{\prime \prime}, 20^{\prime \prime}$, $25^{\prime \prime}$, and $30^{\prime \prime}$. The data were acquired at frequencies spanning $89 \mathrm{GHz}$ to $223 \mathrm{GHz}$, implying beam sizes between $30^{\prime \prime}$ and $11^{\prime \prime}$ (Table 6). The different hypothesis on the source size will then correct differentially for the dilution depending on the frequency of the transition. If the beam size is smaller than the source, no correction is done, whereas dilution has to be corrected for observations with beam sizes that are bigger than the source. Methanol has the specificity of showing no monotonic relation between the upper energy and the frequency of the transition (contrary to $\mathrm{CO}$, for example), and thus the dilution correction has quite an unpredictable effect on the rotational diagram.

For each assumed source size we calculated the reduced $\chi^{2}$ of the linear fit in the rotational diagram. If the assumed source size is the only origin of the scattering in the diagram, this reduced $\chi^{2}$ is minimum when the assumed source size is close to the real source size.

The results of this study are presented in Table 2 . The reduced $\chi^{2}$ decreases when increasing the source size, suggesting that the source is extended. Nevertheless, this whole study rests on three assumptions: the emission is homogeneous on the extent of the source, the source size is the same for all transitions, and scattering in the rotational diagram is only caused by the dilution effect. These three hypotheses may not be valid. Indeed, the different transitions may be emitted by different regions; for instance, the high energy transitions may originate in warmer and less extended regions than do low-lying transitions. The scattering in the diagram may also be caused by opacity or non-LTE effects. It may thus be unrealistic to derive the source size by this method. Only interferometric observations may help to solve this issue.

Nevertheless, it is worth studying the uncertainties led by the source size assumption on the column density of the various isotopomers. Table 2 presents the column densities of methanol isotopomers versus the source size, obtained as follows. For $\mathrm{CH}_{3} \mathrm{OH}$, column densities can only be estimated accurately for sizes of emission smaller than $15^{\prime \prime}$, because no observation was made with a beam larger than $15^{\prime \prime}$. The $\mathrm{CH}_{3} \mathrm{OD}$ and $\mathrm{CHD}_{2} \mathrm{OH}$ column densities were determined using the rotational temperature derived for $\mathrm{CH}_{2} \mathrm{DOH}$, for which we have the largest number of transitions. Column densities change by a factor 2 to 3 according to the size of the source. In contrast, the ratios between isotopomers vary only slightly with the source size (cf. Table 2). In the following, we thus present the column densities assuming a source size of $10^{\prime \prime}$. Finally, care should be taken in the use of the fractionation ratios relative to the main isotopomers $\left(\mathrm{CH}_{3} \mathrm{OH}\right.$ or $\left.\mathrm{H}_{2} \mathrm{CO}\right)$, as these species might be optically 
Table 2. Column densities for methanol isotopomers towards IRAS4A, derived by fixing the rotational temperature to the one derived for $\mathrm{CH}_{2} \mathrm{DOH}$, and ratios between isotopomers.

\begin{tabular}{|c|c|c|c|c|c|c|c|}
\hline $\begin{array}{c}\text { Source } \\
\text { size }\end{array}$ & $\begin{array}{l}T_{\text {rot }} \\
(\mathrm{K})\end{array}$ & $\begin{array}{c}\mathrm{CH}_{2} \mathrm{DOH} \\
\left(\times 10^{14} \mathrm{~cm}^{-2}\right)\end{array}$ & $\chi_{\text {red }}^{2}$ & $\begin{array}{c}\mathrm{CH}_{3} \mathrm{OD} \\
\left(\times 10^{13} \mathrm{~cm}^{-2}\right)\end{array}$ & $\begin{array}{c}\mathrm{CHD}_{2} \mathrm{OH} \\
\left(\times 10^{13} \mathrm{~cm}^{-2}\right)\end{array}$ & $\frac{\mathrm{CH}_{3} \mathrm{OD}}{\mathrm{CH}_{2} \mathrm{DOH}}$ & $\frac{\mathrm{CHD}_{2} \mathrm{OH}}{\mathrm{CH}_{2} \mathrm{DOH}}$ \\
\hline $10^{\prime \prime}$ & $27.1 \pm 1.3$ & $4.3 \pm 0.4$ & 13.5 & $3.1 \pm 0.7$ & $11 \pm 1.7$ & $(7.2 \pm 1.8) \times 10^{-2}$ & $0.26 \pm 0.05$ \\
\hline $15^{\prime \prime}$ & $31.3 \pm 1.7$ & $2.4 \pm 0.3$ & 8.5 & $1.6 \pm 0.4$ & $6.3 \pm 1.0$ & $(6.7 \pm 1.9) \times 10^{-2}$ & $0.26 \pm 0.05$ \\
\hline $20^{\prime \prime}$ & $36.2 \pm 2.3$ & $1.8 \pm 0.2$ & 5.2 & $1.2 \pm 0.3$ & $5.0 \pm 0.9$ & $(6.7 \pm 1.8) \times 10^{-2}$ & $0.28 \pm 0.06$ \\
\hline $25^{\prime \prime}$ & $42.2 \pm 3.2$ & $1.7 \pm 0.2$ & 3.2 & $1.4 \pm 0.3$ & $4.4 \pm 0.8$ & $(8.2 \pm 2.0) \times 10^{-2}$ & $0.26 \pm 0.06$ \\
\hline $30^{\prime \prime}$ & $46.2 \pm 3.8$ & $1.7 \pm 0.2$ & 2.9 & $1.5 \pm 0.4$ & $3.9 \pm 0.7$ & $(8.8 \pm 2.6) \times 10^{-2}$ & $0.23 \pm 0.05$ \\
\hline
\end{tabular}

thick. On the contrary, ratios between deuterated isotopomers are likely not to be affected by such a problem and are thus reliable.

\subsection{Formaldehyde and methanol fractionation}

Figure 1 presents the rotational diagrams derived for the deuterated isotopomers of formaldehyde towards the seven sources of the sample, assuming $10^{\prime \prime}$ source sizes. Table 3 lists the derived rotational temperatures and column densities. For consistency with the analysis of $\mathrm{HDCO}$ and $\mathrm{D}_{2} \mathrm{CO}$, the $\mathrm{H}_{2} \mathrm{CO}$ column density has been recomputed using the rotational diagram method from the data of Maret et al. (2004), with the ortho/para ratio fixed to its statistical value (contrary to the study of Maret et al. (2004) where this ratio was considered as a free parameter), and taking the line opacities into account for the transitions where $\mathrm{H}_{2}{ }^{13} \mathrm{CO}$ has been observed. Note that the column densities inferred by treating the ortho/para ratio as a free parameter are less than a factor 2 different from our values (Maret et al. 2004). The derived rotational temperatures for $\mathrm{HDCO}$ and $\mathrm{D}_{2} \mathrm{CO}$ are in some cases different from the one derived for $\mathrm{H}_{2} \mathrm{CO}$, but it should be noticed that the transitions only span a small interval of upper energies and thus do not constrain the rotational temperature very well. In order to get a sense of the uncertainty implied by these different rotational temperatures, we also computed the HDCO column densities assuming the same rotational temperature as $\mathrm{H}_{2} \mathrm{CO}$ (cf. Table 3). Results were found in most cases to be within a factor of two uncertainties, and the fractionation ratios mostly appear to be consistent within the error bars (cf. Table 5, footnote a).

Figure 2 presents the rotational diagrams of the deuterated forms of methanol for the three sources IRAS4a, IRAS4b, and IRAS2, respectively, again assuming a source size of $10^{\prime \prime}$. The $\mathrm{CH}_{3} \mathrm{OD}$ and $\mathrm{CHD}_{2} \mathrm{OH}$ column densities were computed assuming the same rotational temperature as $\mathrm{CH}_{2} \mathrm{DOH}$, for which many more lines were observed in all sources. As for formaldehyde, the $\mathrm{CH}_{3} \mathrm{OH}$ column density was recomputed from Maret et al. (2005) using rotational diagrams.

\section{Discussion}

In this section, we discuss the results of the observation of deuterated formaldehyde and methanol towards the sample of low-mass class 0 protostars. Table 5 summarizes the observed deuterium fractionations. For comparison, we added the fractionations measured in IRAS 16293 (Loinard et al. 2001; Parise et al. 2002, 2004). Roberts et al. (2002) observed the HDCO fractionation in a number of protostellar cores, including L1448mm and L1527, using the Kitt Peak $12 \mathrm{~m}$ telescope. They found noticeably lower values than ours in these two sources (0.069 and 0.066, respectively). This may come from the fact that the $12 \mathrm{~m}$ telescope beam size is 2.5 times bigger than the $30 \mathrm{~m}$, so that their observations encompass the surrounding cloud where the fractionation is expected to be lower.
The main result of this observational study is the discovery of a high fractionation ratio for formaldehyde and methanol for each source where these molecules were detected. The measured fractionation ratios are indeed similar to those observed in IRAS 16293. In the sources where the isotopes were not detected, the derived upper limits do not exclude a similar deuteration. The present observations confirm that IRAS 16293 is not an exception regarding deuteration. Other studies also confirm that its chemistry is not peculiar. Indeed Bottinelli et al. (2004) observed complex molecules in the hot core of IRAS4A, molecules that were detected for the first time in a low-mass protostar towards IRAS 16293 (Cazaux et al. 2003).

We explore the correlations between the deuteration and other parameters in the following paragraph. We then compare the observed fractionations to the fractionations predicted by grain chemistry models.

\subsection{Correlations}

Figure 3 shows the measured $\mathrm{D}_{2} \mathrm{CO} / \mathrm{H}_{2} \mathrm{CO}$ and $\mathrm{CH}_{2} \mathrm{DOH} / \mathrm{CH}_{3} \mathrm{OH}$ as function of the bolometric luminosity and the $L_{\mathrm{smm}} / L_{\mathrm{bol}}$ (from André et al. 2000). The figures show no obvious correlations between the measured fractionation and these two quantities, despite a variation of one order of magnitude in the $\mathrm{D}_{2} \mathrm{CO} / \mathrm{H}_{2} \mathrm{CO}$ ratio. This is indeed consistent with the hypothesis that the deuteration is a memory of the precollapse phase, rather than a present-day product (e.g. Ceccarelli et al. 2001). If one cannot therefore use the fractionation to distinguish the evolutionary status of the sources, it is likely that the fractionation gives insights into the precollapse phase of each source.

Figure 4 presents the $\mathrm{D}_{2} \mathrm{CO} / \mathrm{H}_{2} \mathrm{CO}$ fractionation ratio (as well as the $\mathrm{D}_{2} \mathrm{CO} / \mathrm{HDCO}$ ratio in order to get rid of any potential opacity effect) versus the $\mathrm{CO}$ depletion in the sources of our sample. The $\mathrm{CO}$ depletion was computed from the $\mathrm{CO}$ densities in the outer envelope derived by Jørgensen et al. (2002), and it assumes a canonical CO abundance of $8.4 \times 10^{-5}$ (Frerking et al. 1982). We also plotted in this figure the result for prestellar cores observed by Bacmann et al. (2002).

The first thing to notice is that Class 0 sources and prestellar cores with similar $\mathrm{CO}$ depletion factors also present similar $\mathrm{D}_{2} \mathrm{CO} / \mathrm{H}_{2} \mathrm{CO}$ ratios. This is another suggestion that the deuteration of formaldehyde in Class 0 sources occurred during the precollapse phase and/or that the physical conditions in the outer envelopes of Class 0 sources are very similar to prestellar cores. On the other hand, and surprising enough, the two sources with the highest $\mathrm{D}_{2} \mathrm{CO} / \mathrm{H}_{2} \mathrm{CO}$ ratio are those with the lowest $\mathrm{CO}$ depletion factor. Indeed, chemical models (e.g. Roberts \& Millar 2000) predict that formaldehyde fractionation should increase with $\mathrm{CO}$ depletion. However, we investigate in the following if this could be caused by the optical thickness of $\mathrm{H}_{2} \mathrm{CO}$, leading to artificially high $\mathrm{D}_{2} \mathrm{CO} / \mathrm{H}_{2} \mathrm{CO}$ ratios. 


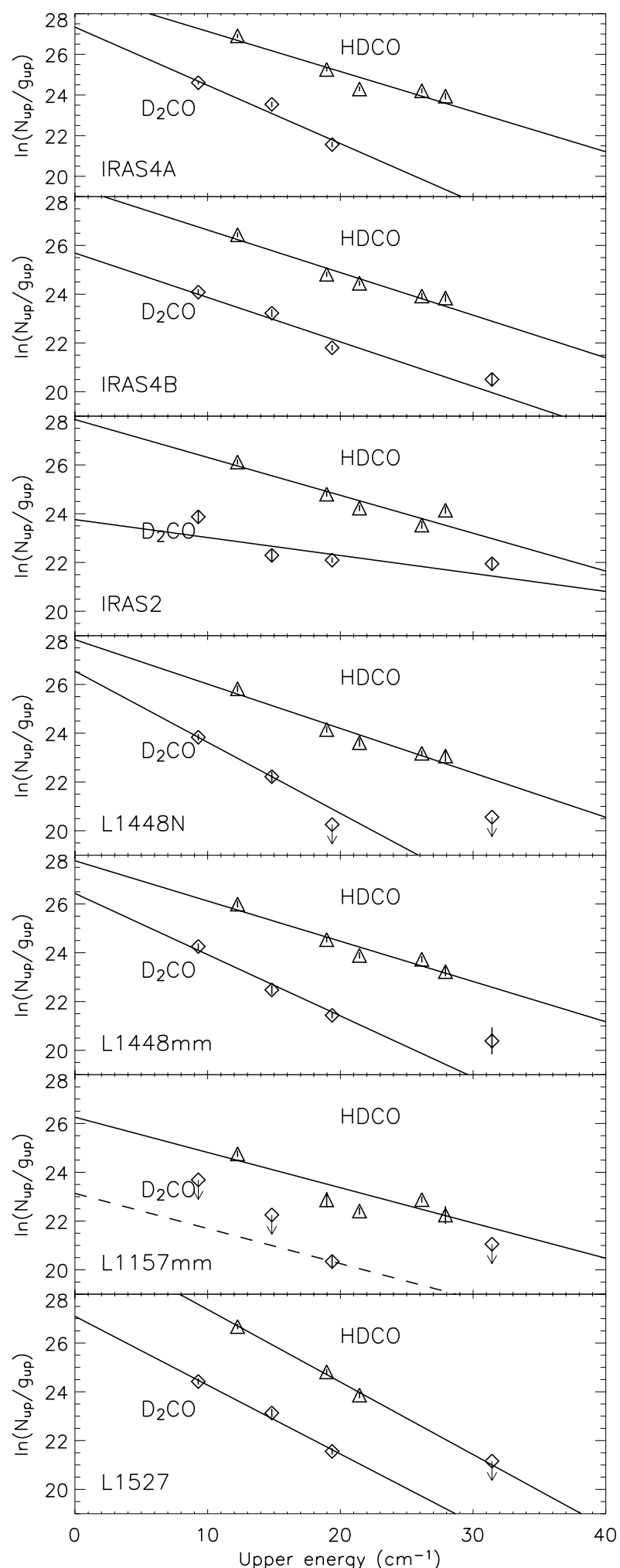

Fig. 1. Rotational diagrams for deuterated formaldehyde for the seven studied sources, assuming 10" for the source size. Error bars correspond to the error bars on the flux as stated in Tables 6-8.

Both sources (L1527 and L1448mm) show evidence of very optically thick $\mathrm{CO}$ emission. A study of $\mathrm{C}^{18} \mathrm{O}$ and $\mathrm{C}^{17} \mathrm{O}$ emission (Jørgensen et al. 2002) towards a sample including all our sources shows that L1527 and L1448mm are the sources for which $\mathrm{C}^{18} \mathrm{O}$ is the most optically thick (Jørgensen et al. 2002). Thus $\mathrm{H}_{2} \mathrm{CO}$ is more likely to be optically thick

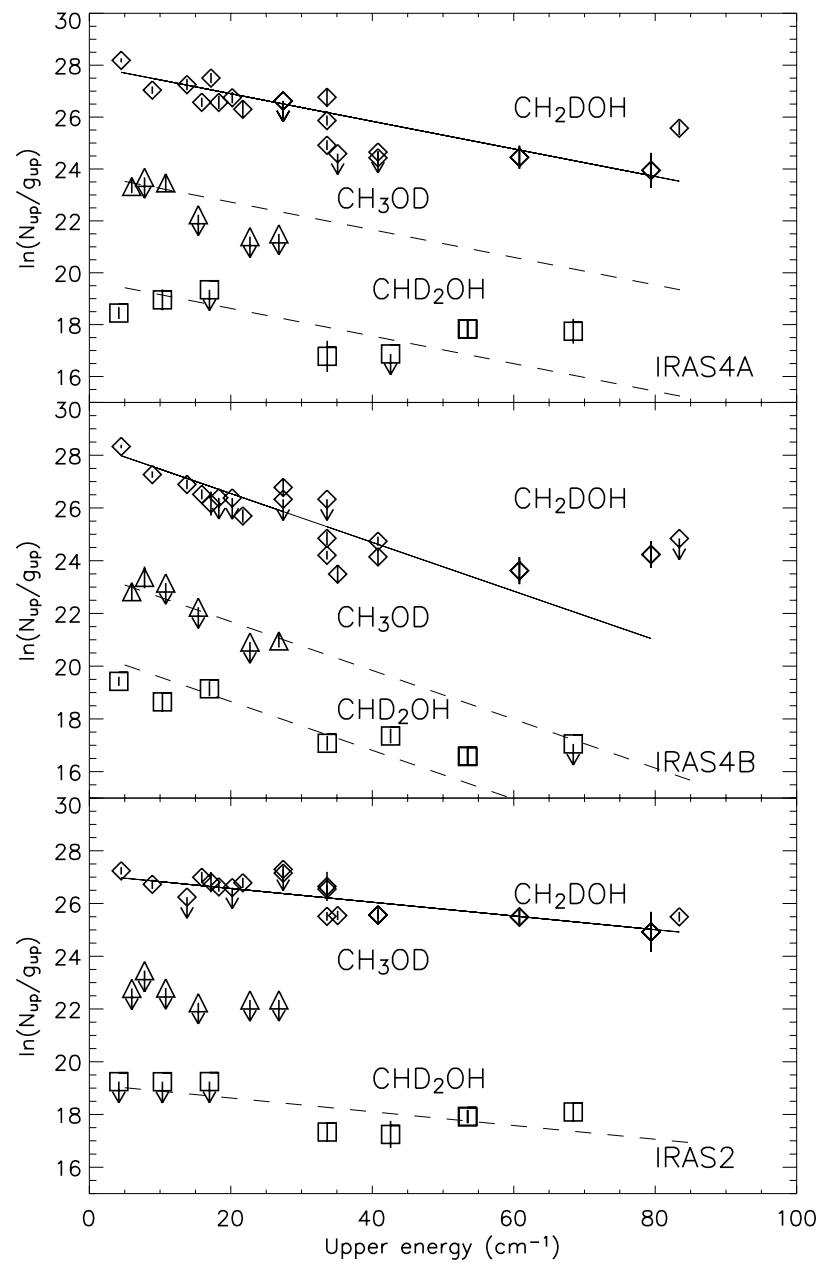

Fig. 2. Rotational diagrams for deuterated methanol in IRAS4A, IRAS4B and IRAS2 (for the sake of clarity the curves for $\mathrm{CH}_{3} \mathrm{OD}$ and $\mathrm{CHD}_{2} \mathrm{OH}$ have been translated by -2 and -7 along the $y$-axis). Error bars correspond to the error bars on the flux as stated in Tables 6 and 7. Solid lines $\left(\mathrm{CH}_{2} \mathrm{DOH}\right)$ correspond to fits to the data, with two free parameters $\left(T_{\text {rot }}\right.$ and $\left.N_{\text {tot }}\right)$. Dashed lines correspond to fits with only $N_{\text {tot }}$ as a free parameter, and $T_{\text {rot }}$ set to the value derived for $\mathrm{CH}_{2} \mathrm{DOH}$ (cf. text).

(as suggested by the correlation between the $\mathrm{H}_{2} \mathrm{CO}$ and CO abundances, Maret et al. 2004). Maret et al. (2004) observed some transitions from $\mathrm{H}_{2}^{13} \mathrm{CO}$ in a few sources. Unfortunately, no line was observed for L1527, and only an upper limit was derived for one line on $\mathrm{L} 1448 \mathrm{~mm}(\tau<2)$. In the case of L1448mm, we thus might have underestimated the $\mathrm{H}_{2} \mathrm{CO}$ column density by a factor as high as $\frac{\tau}{1-e^{\tau}} \sim 2.3$. For L1527, the opacity is likely to be even larger as suggested by the $\mathrm{C}^{18} \mathrm{O} / \mathrm{C}^{17} \mathrm{O}$ measured by Jørgensen et al. (2002). However, we conclude from this study that this opacity effect cannot fully account for the discrepancy of 2 orders of magnitude observed in $\mathrm{D}_{2} \mathrm{CO} / \mathrm{H}_{2} \mathrm{CO}$ compared to prestellar cores. Indeed, the $\mathrm{D}_{2} \mathrm{CO} / \mathrm{HDCO}$, which is supposed to be less affected by opacity effects than the ratio with the main isotopomer, is still too high for one of the sources (L1448mm). Hence, the conclusion is that, while there seems to be a correlation between $\mathrm{CO}$ depletion and fractionation in prestellar cores, no such correlation is apparent in our sample of YSO (no correlation is found either between the fractionation and the $\mathrm{N}_{2} \mathrm{H}^{+}$ abundance, tracing the $\mathrm{N}_{2}$ depletion). This difference may imply 
that the CO depletion in the circumstellar envelopes is affected by outgassing due to the heating by the newly formed star.

Finally, our analysis, based on rotational diagrams, allows only an estimation of the fractionation averaged on the beam. It will be important to understand what the contribution of the different regions (warm envelope, cold envelope, etc) is. New insights in this respect may only be possible by interferometric observations, to observe the relative fractionation of formaldehyde and methanol in the two regions: hot core and outer envelope.

\subsection{Comparison to grain chemistry models}

Grain surface chemistry is thought to be responsible for the formation of the abundant methanol observed in hot cores for two main reasons. First, gas-phase chemistry models fail to reproduce the $\mathrm{CH}_{3} \mathrm{OH}$ abundance by 2 to 3 orders of magnitude in Orion (Menten et al. 1988). Recent investigations have confirmed that no gas-phase process can efficiently form methanol (Herbst 2005). Second, methanol is one of the most abundant species observed in the grain mantles (after water), with abundances as high as $30 \%$ of the water abundance (Dartois et al. 1999).

Methanol is thought to be formed on the grains by successive hydrogenations of $\mathrm{CO}$. An intermediate product of these reactions is formaldehyde. Deuterium fractionation studies of formaldehyde and methanol thus provide a useful tool for confirming the grain chemistry scenario. To understand if formaldehyde and methanol are formed simultanuously on the grains and to unveil a possible contribution of gas-phase processes to the formation of formaldehyde, we compared the observed fractionations to the predictions of the grain chemistry model from Stantcheva \& Herbst (2003). This model is based on the resolution of the rate equations governing the abundance of each species on the grain, and it uses the master equation approach in the cases where only a small number of molecules are present on the surface.

Figure 5 presents the $\mathrm{HDCO}, \mathrm{D}_{2} \mathrm{CO}, \mathrm{CH}_{2} \mathrm{DOH}, \mathrm{CH}_{3} \mathrm{OD}$, and $\mathrm{CHD}_{2} \mathrm{OH}$ fractionations predicted by the grain model as a function of the gas-phase atomic $\mathrm{D} / \mathrm{H}$ ratio at the time of mantle formation. The observed fractionations with their error bars have been superimposed for each source. This allows us to infer the required $\mathrm{D} / \mathrm{H}$ ratio required for the formation of each molecule. For comparison, Fig. 5 also presents the same study for IRAS 16293 (Ceccarelli et al. 1998; Loinard et al. 2000; Parise et al. 2004).

A comparison between formaldehyde and methanol must be made with care as it is very dependent on the possible opacity of the main isotopomers $\mathrm{H}_{2} \mathrm{CO}$ and $\mathrm{CH}_{3} \mathrm{OH}$. In contrast, a comparison between different deuterated isotopomers of methanol should not suffer from such problem. We attribute to this opacity issue the fact that, depending on the source, either HDCO or $\mathrm{D}_{2} \mathrm{CO}$ is compatible with methanol fractionation.

\section{Several conclusions can be drawn from this comparison:}

- Whenever they are correctly constrained, the $\mathrm{CH}_{2} \mathrm{DOH}$ and $\mathrm{CHD}_{2} \mathrm{OH}$ fractionations are consistent, i.e. they have a common range of possible atomic D/H ratio (IRAS 16293, IRAS4A, IRAS4B, and IRAS2). That had already been observed for IRAS 16293 (Parise et al. 2002, 2004), for which $\mathrm{CD}_{3} \mathrm{OH}$ fractionation was also consistent with the latter two isotopomers. The present, new observations thus further support the hypothesis that methanol is formed on the grain surfaces.
- Whenever it is contrained, $\mathrm{CH}_{3} \mathrm{OD}$ appears to be underabundant with respect to the other methanol isotopomers. This had already been noticed for the case of IRAS 16293 by Parise et al. (2002), who concluded that $\mathrm{CH}_{3} \mathrm{OD}$ may be destroyed in the gas phase by protonation more rapidly than the other isotopomers, owing to the strong electronegativity of the oxygen atom. This conclusion was also suggested by the theoretical study of Osamura et al. (2004). However, some very recent laboratory experiments suggest another possibility. Indeed, Nagaoka et al. (2005) show that deuterated methanol species with an OD bond are very inefficiently formed on ice surfaces when exposing $\mathrm{CH}_{3} \mathrm{OH}$ to $\mathrm{H}$ and $\mathrm{D}$ atoms, while isotopomers with $\mathrm{CD}$ bonds are abundantly formed. This might also explain the non-detection of HDO in ices of protostellar envelopes (Dartois et al. 2003; Parise et al. 2003) and the fractionation of water observed to be one order of magnitude lower than $\mathrm{CH}_{2} \mathrm{DOH} / \mathrm{CH}_{3} \mathrm{OH}$ in the hot corino of IRAS 16293 , in agreement with these solid state observations (Parise et al. 2005).

- HDCO appears to be less abundant than $\mathrm{D}_{2} \mathrm{CO}$ with respect to the model predictions (except for L1157mm, but for this source, the error bars are important). No preferential destruction in the gas phase could explain this discrepancy (as was the case for $\mathrm{CH}_{3} \mathrm{OD}$ ). This might be caused by $\mathrm{H}-\mathrm{D}$ substitution via hydrogen abstraction reactions $\left(\mathrm{HDCO}+\mathrm{H} \rightarrow \mathrm{DCO}+\mathrm{H}_{2}\right.$ ), which are not included in this chemistry model. These reactions would favor the formation of $\mathrm{D}_{2} \mathrm{CO}$ by abstracting an hydrogen to HDCO and deuterating DCO (Tielens 1983). Alternatively, this might be a hint that formaldehyde formation and fractionation are not dominated by grain chemistry processes. We discuss the gas-phase formation and fractionation of formaldehyde more thoroughly in the next section.

\subsection{Gas-phase formation of formaldehyde}

As discussed in the previous section, there is now good evidence that methanol is formed on the grains, and the data of this survey support this hypothesis further (as presented in the previous paragraph). Yet, the situation is not so clear for formaldehyde. After discussing the spatial origin of the $\mathrm{HDCO}$ and $\mathrm{D}_{2} \mathrm{CO}$ emission, we examine the implications of the present observations on the formaldehyde formation process.

In contrast to methanol that shows quite high rotational temperatures, and thus may be arising in the hot corinos, deuterated formaldehyde shows low rotational temperatures, instead pointing to an origin in the cold envelope of the protostars. The study by Ceccarelli et al. (2001) of the distribution of the $\mathrm{D}_{2} \mathrm{CO} \quad 4_{0,4}-3_{0,3}$ line $\left(E_{\mathrm{up}}=19.4 \mathrm{~cm}^{-1}\right.$, a rather low energy line) towards IRAS 16293-2422 shows that it is extended over the entire envelope and that the $\mathrm{D}_{2} \mathrm{CO} / \mathrm{H}_{2} \mathrm{CO}$ ratio is also high across the envelope. These authors also noticed that while the $\mathrm{D}_{2} \mathrm{CO}$ emission correlates well with the continuum emission tracing the envelope, it does not show any enhancement in correspondence with the shock sites in the region caused by the interface between the outflow and the envelope itself. They concluded that $\mathrm{D}_{2} \mathrm{CO}$ is abundant in the cold outer envelope, where the dust temperature is lower than $100 \mathrm{~K}$. Of course, this does not exclude the possibility of abundant $\mathrm{D}_{2} \mathrm{CO}$ also in the hot corino region, but the available observations were unable to detect it. Note that Fuente et al. (2005) found, from inferferometric observations, abundant $\mathrm{D}_{2} \mathrm{CO}$ in the hot core of the intermediate mass protostar NGC 7129-FIRS2. As in the case of IRAS 16293-2422, the present observations are unable to disentangle any 
Table 3. $\mathrm{H}_{2} \mathrm{CO}$, HDCO, and $\mathrm{D}_{2} \mathrm{CO}$ rotational temperatures and column densities, assuming a $10^{\prime \prime}$ source.

\begin{tabular}{cccccccc}
\hline \hline Source & \multicolumn{2}{c}{$\mathrm{H}_{2} \mathrm{CO}^{a}$} & \multicolumn{3}{c}{$\mathrm{HDCO}$} \\
& $\begin{array}{c}T_{\text {rot }} \\
(\mathrm{K})\end{array}$ & $\begin{array}{c}N_{\text {tot }} \\
\left(\times 10^{14} \mathrm{~cm}^{-2}\right)\end{array}$ & $\begin{array}{c}T_{\text {rot }} \\
(\mathrm{K})\end{array}$ & $\begin{array}{c}N_{\text {tot }} \\
\left(\times 10^{13} \mathrm{~cm}^{-2}\right)\end{array}$ & $\begin{array}{c}N_{\text {tot }}^{b} \\
\left(\times 10^{13} \mathrm{~cm}^{-2}\right)\end{array}$ & $\begin{array}{c}T_{\text {rot }} \\
(\mathrm{K})\end{array}$ & $\begin{array}{c}N_{\text {tot }} \\
\left(\times 10^{12} \mathrm{~cm}^{-2}\right)\end{array}$ \\
\hline IRAS4A & $20 \pm 2$ & $1.7 \pm 0.4$ & $7.3 \pm 0.3$ & $3.2 \pm 0.6$ & $1.2 \pm 0.2$ & $5.0 \pm 0.3$ & $19 \pm 4$ \\
IRAS4B & $38 \pm 6$ & $1.7 \pm 0.5$ & $8.2 \pm 0.5$ & $1.9 \pm 0.4$ & $1.2 \pm 0.3$ & $7.9 \pm 0.5$ & $7.0 \pm 1.5$ \\
IRAS2 & $20 \pm 2$ & $0.84 \pm 0.22$ & $9.3 \pm 0.6$ & $1.3 \pm 0.3$ & $0.82 \pm 0.13$ & $19.6 \pm 3.6$ & $4.0 \pm 1.5$ \\
L1448N & $14 \pm 1$ & $1.1 \pm 0.2$ & $7.9 \pm 0.5$ & $1.0 \pm 0.3$ & $0.46 \pm 0.057$ & $4.9 \pm 0.8$ & $8.2 \pm 4.4$ \\
L1448mm & $16 \pm 1$ & $0.42 \pm 0.11$ & $8.7 \pm 0.6$ & $1.1 \pm 0.3$ & $0.57 \pm 0.063$ & $5.7 \pm 0.3$ & $9.1 \pm 2.3$ \\
L1157mm & $12 \pm 1$ & $0.20 \pm 0.06$ & $10.0 \pm 0.9$ & $0.29 \pm 0.09$ & $0.24 \pm 0.034$ & - & $\leq 1.5$ \\
L1527 & $12 \pm 1$ & $0.42 \pm 0.17$ & $4.8 \pm 0.3$ & $6.0 \pm 1.8$ & $1.1 \pm 0.16$ & $5.1 \pm 0.3$ & $14.9 \pm 4.2$ \\
\hline
\end{tabular}

${ }^{a}$ Reanalysis of the data from Maret et al. (2004) with the rotational diagram method (cf. text). ${ }^{b}$ Assuming the same rotational temperature as $\mathrm{H}_{2} \mathrm{CO}$.

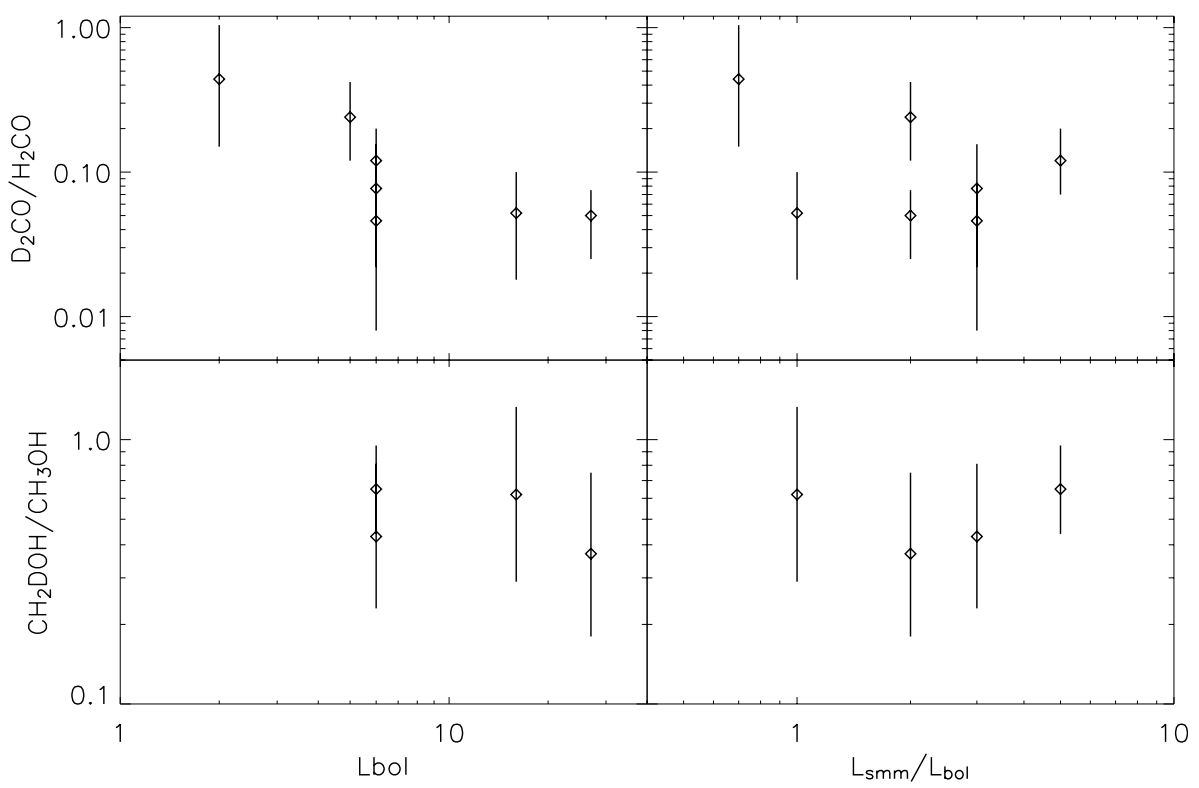

Fig. 3. Fractionation of formaldehyde and methanol as a function of bolometric luminosity and the $L_{\mathrm{smm}} / L_{\mathrm{bol}}$ (from André et al. 2000).

Table 4. Methanol rotational temperatures and column densities, assuming a 10" source size.

\begin{tabular}{|c|c|c|c|c|c|c|}
\hline \multirow[t]{2}{*}{ Source } & \multicolumn{2}{|c|}{$\overline{\mathrm{C} \mathrm{CH}_{3} \mathrm{OH}}$} & \multicolumn{2}{|c|}{$\overline{\mathrm{CH}_{2} \mathrm{DOH}}$} & \multirow{2}{*}{$\begin{array}{c}\mathrm{CH}_{3} \mathrm{OD} \\
N_{\text {tot }} \\
\left(\times 10^{13} \mathrm{~cm}^{-2}\right)\end{array}$} & \multirow{2}{*}{$\begin{array}{c}\mathrm{CHD}_{2} \mathrm{OH} \\
N_{\text {tot }} \\
\left(\times 10^{14} \mathrm{~cm}^{-2}\right)\end{array}$} \\
\hline & $\begin{array}{l}T_{\text {rot }} \\
(\mathrm{K}) \\
\end{array}$ & $\begin{array}{c}N_{\text {tot }} \\
\left(\times 10^{14} \mathrm{~cm}^{-2}\right)\end{array}$ & $\begin{array}{l}T_{\text {rot }} \\
(\mathrm{K})\end{array}$ & $\begin{array}{c}N_{\text {tot }} \\
\left(\times 10^{14} \mathrm{~cm}^{-2}\right)\end{array}$ & & \\
\hline IRAS 4A & $38.0 \pm 3.2$ & $6.9 \pm 1.4$ & $27.1 \pm 1.3$ & $4.3 \pm 0.4$ & $3.1 \pm 0.7^{c}$ & $1.1 \pm 0.17^{c}$ \\
\hline IRAS 4B & $84.9 \pm 16.8$ & $8.0 \pm 2.7$ & $15.6 \pm 0.5$ & $2.9 \pm 0.2$ & $1.1 \pm 0.20^{c}$ & $0.90 \pm 0.10^{c}$ \\
\hline IRAS 2 & $207 \pm 48.1$ & $10.1 \pm 3.7$ & $55.3 \pm 4.9$ & $5.2 \pm 0.8$ & $\leq 8.0$ & $2.1 \pm 0.4^{c}$ \\
\hline L1448N & $27.5 \pm 4.5$ & $1.2 \pm 0.47$ & $27.5 \pm 4.5^{a}$ & $2.1 \pm 0.6^{b}$ & $\leq 8.0$ & $\leq 10$ \\
\hline L1448mm & $76.3 \pm 19.1$ & $1.6 \pm 0.70$ & $76.3 \pm 19.1^{a}$ & $11.3 \pm 4.7^{b}$ & $40 \pm 16^{b}$ & $\leq 6.3$ \\
\hline $\mathrm{L} 1157 \mathrm{~mm}$ & $112 \pm 201$ & $1.9 \pm 5.2$ & $112 \pm 201^{a}$ & $10.3 \pm 27.7^{b}$ & $22 \pm 66^{b}$ & $\leq 9.6$ \\
\hline
\end{tabular}

${ }^{a}$ Temperature fixed to the rotational temperature of $\mathrm{CH}_{3} \mathrm{OH}$.

${ }^{b}$ Should be taken as an upper limit, as the rotational temperature is likely to be overestimated.

${ }^{c}$ Temperature fixed to the rotational temperature of $\mathrm{CH}_{2} \mathrm{DOH}$.

possible contribution from the hot corino, as well as from the shocks around these sources, but cannot exclude these contributions either. Given the uncertainty on the location of the $\mathrm{D}_{2} \mathrm{CO}$, we analyze both possible situations in the following, hot corino versus cold envelope.

The observed high deuterium fractionation of formaldehyde (Ceccarelli et al. 1998; Loinard et al. 2001, and this paper), if arising in the hot corino, excludes an efficient production of formaldehyde in the warm gas, for which a much lower deuteration would be expected. Hence, these high formaldehyde abundance and fractionation must reflect the cold phase preceeding the hot-core phase accompanying the formation of the protostar. Gas phase models typically produce formaldehyde abundance that ranges from about $10^{-6}$ at early times to $10^{-8}$ at late times (e.g. in steady state). Abundance observed in cold dark clouds is typically $10^{-8}$ and in good agreement with the latter value. However, abundances are observed to be higher in the hot corino around protostars $\left(\sim 10^{-7}-10^{-6}\right.$, Maret et al. 2004, 2005), so models based upon gas phase production of the formaldehyde in hot cores have to selectively deplete the formaldehyde in order 
Table 5. Deuterium fractionation for formaldehyde and methanol, for an assumed source size of $10^{\prime \prime}$. Error bars are associated with a $90 \%$ confidence interval (see appendix).

\begin{tabular}{|c|c|c|c|c|c|c|c|}
\hline Source & ${\frac{\mathrm{HDCO}}{\mathrm{H}_{2} \mathrm{CO}}}^{a}$ & $\frac{\mathrm{D}_{2} \mathrm{CO}}{\mathrm{H}_{2} \mathrm{CO}}$ & $\frac{\mathrm{D}_{2} \mathrm{CO}}{\mathrm{HDCO}}$ & $\frac{\mathrm{CH}_{2} \mathrm{DOH}}{\mathrm{CH}_{3} \mathrm{OH}}$ & $\frac{\mathrm{CH}_{3} \mathrm{OD}}{\mathrm{CH}_{3} \mathrm{OH}}$ & $\frac{\mathrm{CHD}_{2} \mathrm{OH}}{\mathrm{CH}_{3} \mathrm{OH}}$ & $\frac{\mathrm{CHD}_{2} \mathrm{OH}}{\mathrm{CH}_{2} \mathrm{DOH}}$ \\
\hline IRAS4A & $0.20_{-0.09}^{+0.13}$ & $0.12_{-0.05}^{+0.08}$ & $0.62_{-0.26}^{+0.33}$ & $0.65_{-0.21}^{+0.30}$ & $0.047_{-0.021}^{+0.029}$ & $0.17_{-0.06}^{+0.09}$ & $0.26_{-0.07}^{+0.08}$ \\
\hline IRAS4B & $0.13_{-0.06}^{+0.10}$ & $0.046_{-0.024}^{+0.038}$ & $0.39_{-0.17}^{+0.23}$ & $0.43_{-0.20}^{+0.38}$ & $0.016_{-0.008}^{+0.016}$ & $0.13_{-0.07}^{+0.12}$ & $0.31_{-0.06}^{+0.07}$ \\
\hline IRAS2 & $0.17_{-0.08}^{+0.12}$ & $0.052_{-0.034}^{+0.048}$ & $0.33_{-0.21}^{+0.28}$ & $0.62_{-0.33}^{+0.71}$ & $\leq 0.08$ & $0.25_{-0.14}^{+0.29}$ & $0.41_{-0.15}^{+0.19}$ \\
\hline L1448N & $0.094_{-0.050}^{+0.062}$ & $0.077_{-0.069}^{+0.079}$ & $0.93_{-0.83}^{+1.14}$ & $\leq 1.8$ & $\leq 0.67$ & $\leq 8.3$ & - \\
\hline $\mathrm{L} 1448 \mathrm{~mm}$ & $0.29_{-0.15}^{+0.23}$ & $0.24_{-0.12}^{+0.18}$ & $0.91_{-0.48}^{+0.73}$ & $\leq 7.1$ & $\leq 2.5$ & $\leq 3.9$ & - \\
\hline L1157mm & $0.16_{-0.10}^{+0.15}$ & $\leq 0.08$ & $\leq 0.52$ & $\leq 5.4$ & $\leq 1.2$ & $\leq 5.1$ & - \\
\hline L1527 & $1.7_{-1.1}^{+2.6}$ & $0.44_{-0.29}^{+0.60}$ & $0.28_{-0.16}^{+0.25}$ & - & - & - & - \\
\hline IRAS 16293 & $0.15 \pm 0.07$ & $0.05 \pm 0.025$ & $0.3 \pm 0.2$ & ${ }^{b} 0.37_{-0.19}^{+0.38}$ & ${ }^{b} 0.018_{-0.012}^{+0.022}$ & ${ }^{b} 0.074_{-0.044}^{+0.084}$ & ${ }^{b} 0.21_{-0.10}^{+0.11}$ \\
\hline
\end{tabular}

${ }^{a} \mathrm{HDCO} / \mathrm{H}_{2} \mathrm{CO}$ ratios obtained when assuming the same rotational temperature for $\mathrm{HDCO}$ as for $\mathrm{H}_{2} \mathrm{CO}$ are resp. $0.075_{-0.031}^{+0.047}, 0.080_{-0.044}^{+0.068}, 0.11_{-0.04}^{+0.07}$, $0.043_{-0.015}^{+0.021}, 0.15_{-0.06}^{+0.10}, 0.14_{-0.06}^{+0.11}, 0.33_{-0.18}^{+0.42}$ for the 7 sources.

$\mathrm{HDCO} / \mathrm{H}_{2} \mathrm{CO}$ ratios obtained assuming a $15^{\prime \prime}$ source are resp. $0.14_{-0.07}^{+0.11}, 0.07_{-0.05}^{+0.09}, 0.14_{-0.07}^{+0.11}, 0.08_{-0.04}^{+0.06}, 0.23_{-0.14}^{+0.19}, 0.19_{-0.12}^{+0.22}, 0.89_{-0.63}^{+1.43}$. They are thus consistent with the ratios derived for a $10^{\prime \prime}$ source, within the error bars.

${ }^{b}$ Error bars recomputed from Parise et al. (2004) for a 90\% confidence interval (see appendix).

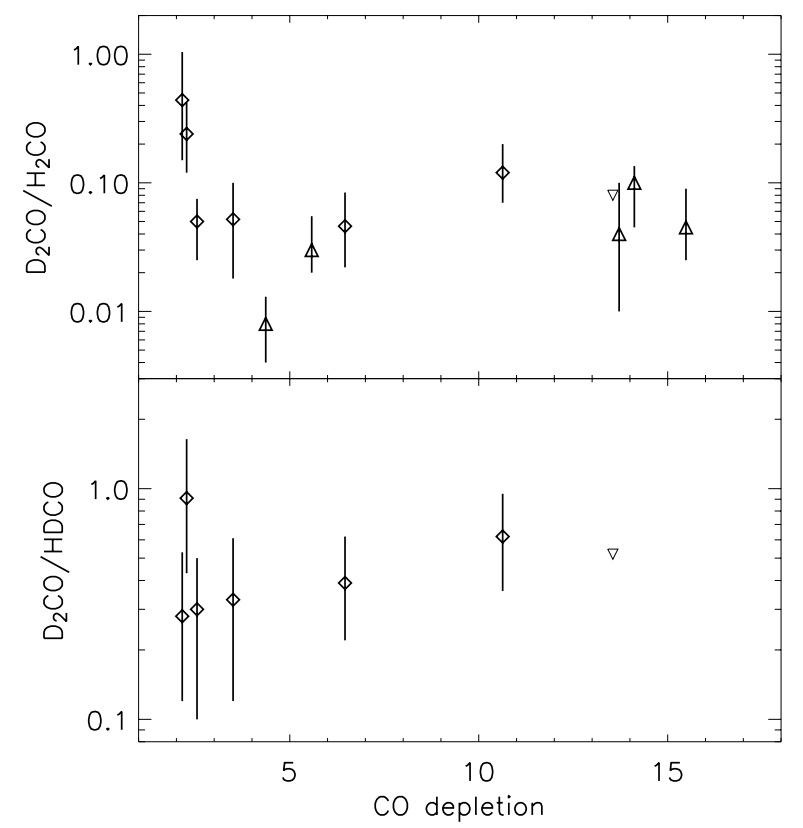

Fig. 4. Doubly-deuteration of formaldehyde versus CO depletion. Class 0 sources (this work) appear as diamonds and prestellar cores (Bacmann et al. 2002) as triangles. The reversed triangle is the upper limit for L1157mm (this work).

to keep the abundance in the dark phase low. While all species are expected to freeze out equally efficiently at the low temperature of dust grains in dark clouds, $\mathrm{CO}$ is perhaps returned to the gas phase by evaporation but formaldehyde is left behind. Then, in order to increase the formaldehyde abundance by a factor of 100 , the $\mathrm{CO}$ has to cycle a hundred times between the gas phase and the ices. This seems a bit contrived. The only gas phase possibility therefore seems formation at early times, when $\mathrm{H}_{2} \mathrm{CO}$ abundance is $10^{-6}$, and immediate depletion and cold storage in unreactive ices. This seems to contradict the depletion studies of dark cloud cores (Tafalla et al. 2002). Furthermore, the bulk of the formaldehyde would be formed under undepleted conditions, so the $\mathrm{D}_{2} \mathrm{CO}$ and HDCO fractionation is difficult to understand (Roberts et al. 2003).

We now study the implications of our observations if the $\mathrm{HDCO}$ and $\mathrm{D}_{2} \mathrm{CO}$ originate mainly in the cold envelope. In this case, we must inquire whether gas phase processes at present times can account for their formation. The discrepancy of our fractionation observations for formaldehyde with the grain chemistry models (namely the fact that HDCO seems to be steadily underabundant compared to $\mathrm{D}_{2} \mathrm{CO}$ ) might indeed reveal that the formation of formaldehyde is not dominated by $\mathrm{H}$ and $\mathrm{D}$ additions to $\mathrm{CO}$ on grain surfaces. In this cold gas, the absolute $\mathrm{H}_{2} \mathrm{CO}$ abundance is explained well by gas-phase models. Models of formaldehyde fractionation in prestellar cores whose chemical and physical conditions are similar to those in the outer cold envelopes of Class 0 sources (Maret et al. 2004; Jørgensen et al. 2005) - seem to approximately reproduce the observed ratios (Roberts et al. 2004). However, the predictions depend on some key reactions, whose rates, products, and branching ratios are relatively uncertain; and recent studies seem to show that gas-phase models may have more difficulty in reproducing the high deuteration than expected. Indeed, Osamura et al. (2005) recently presented quantum chemical calculations of the protonation/deuteration of $\mathrm{H}_{2} \mathrm{CO}$ and deuterated isotopologues. These computations show that the deuteration of $\mathrm{H}_{2} \mathrm{CO}$ by $\mathrm{H}_{3}^{+}$and deuterated isotopomers will mainly happen on the oxygen end of the molecule, due to the higher electronegativity of oxygen. It is generally expected that the dissociative electron recombination of $\mathrm{H}_{2} \mathrm{COD}^{+}$will not lead to the formation of $\mathrm{HDCO}$, so that the fractionation due to reactions of $\mathrm{H}_{2} \mathrm{CO}$ with $\mathrm{H}_{2} \mathrm{D}^{+}$and other isotopomers is quite inefficient. However, given the recent surprising experimental results on the product distribution in the dissociative recombination reaction of $\mathrm{N}_{2} \mathrm{H}^{+}$and $\mathrm{CH}_{3} \mathrm{OH}_{2}^{+}$(Geppert et al. 2006), an experimental verification of the product distribution of this key reaction $\left(\mathrm{H}_{2} \mathrm{COD}^{+}+\mathrm{e}^{-}\right)$would be very valuable. Another possibility in this context is that $\mathrm{H}_{2} \mathrm{CO}$ is formed in the gas-phase and then frozen out on dust grains where it can later be fractionated by abstraction reactions on the grain surfaces. Some efficient desorption processes are then required to release the molecules back to the gas phase. 


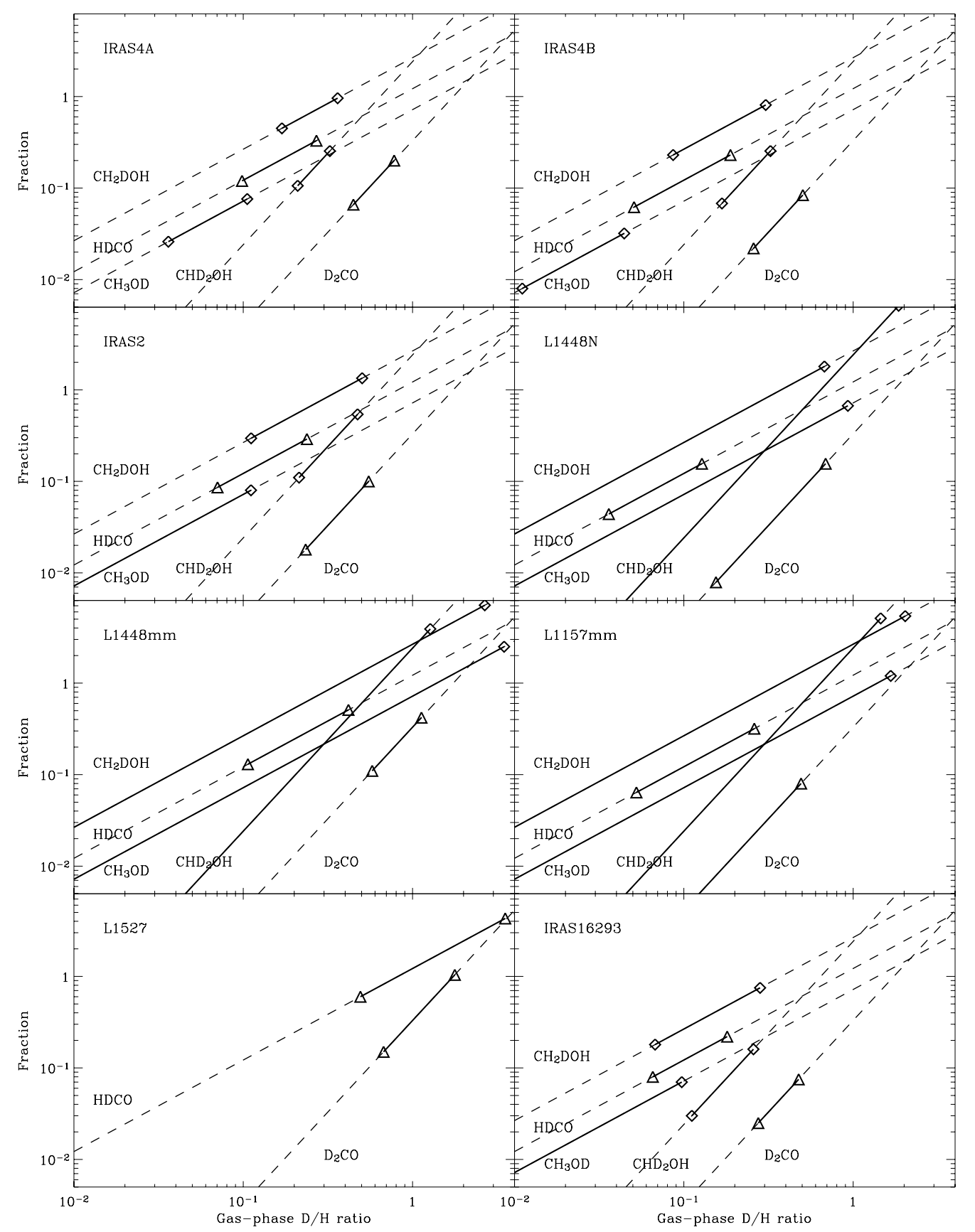

Fig. 5. Comparison between the observed fractionations (triangles are for formaldehedyde species, whereas diamonds are for methanol species) towards the seven sources in our sample (solid lines) and the predictions of the grain model (dashed lines, Stantcheva \& Herbst 2003). IRAS 16293 has been added in the last panel for comparison.

In summary, pure gas-phase models seem to face problems in explaining both formaldehyde absolute abundance and fractionation at the same time. Unless the dissociative recombination of $\mathrm{H}_{2} \mathrm{COD}^{+}$leads to the formation of HDCO, H-D substitutions on grains seem to be required to account for the high fractionation if formaldehyde is formed by gas-phase processes.

\section{Conclusion}

We have presented observations of deuterated formaldehyde and methanol towards a sample of low-mass protostars. We detected $\mathrm{HDCO}, \mathrm{D}_{2} \mathrm{CO}$, and $\mathrm{CH}_{2} \mathrm{DOH}$ in all sources. However, $\mathrm{CH}_{3} \mathrm{OD}$ and $\mathrm{CHD}_{2} \mathrm{OH}$ were only detected towards 2 and 3 sources, respectively. We analyzed the data using population diagrams.
These observations show that IRAS $16293-2422$ is not an exception concerning deuteration. The fractionations derived towards the source of our sample are indeed similar to those observed in IRAS 16293.

These observations are useful for pinning down the contributions of grain surface and gas phase chemistry in the formation of formadehyde and methanol. It appears that methanol fractionation is consistent with grain surface schemes, whereas formaldehyde formation cannot be fully constrained from these data. Two possibilities arise, either that formaldehyde formation is dominated by gas-phase reactions and later fractionated on the grains through abstraction reactions or that formaldehyde is formed on the grains, with abstraction reactions altering the expected $\mathrm{D}_{2} \mathrm{CO} / \mathrm{HDCO}$ ratio. 


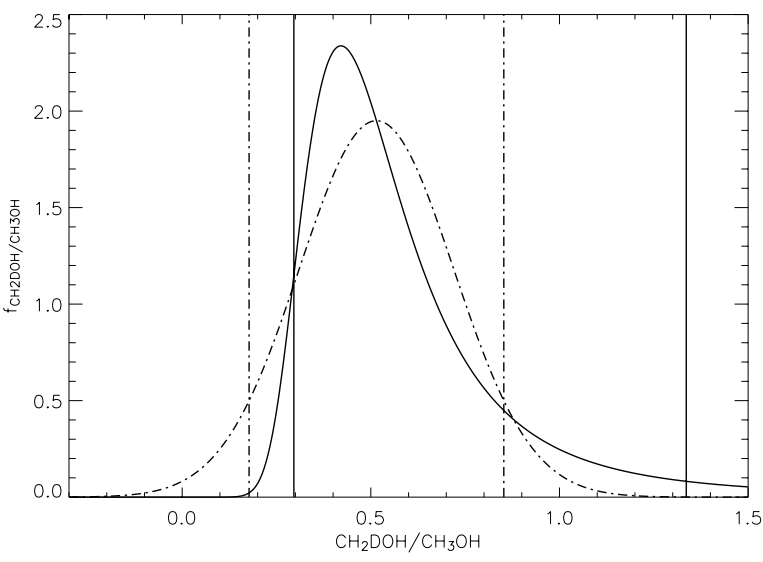

Fig. 6. Repartition function for the $\mathrm{CH}_{2} \mathrm{DOH} / \mathrm{CH}_{3} \mathrm{OH}$ ratio (solid line). Overlaid in a dash-dot line is the repartition function computed assuming that the ratio is Gaussian, with a width computed by the standard error propagation. Vertical lines delimitate the $90 \%$ confidence intervals for the two distributions.

This study is limited by different factors. All the transitions were observed with different beam sizes, with a best spatial resolution of $10^{\prime \prime}$. It is very difficult in this case to derive the abundance of the molecules if one does not know the source size. Moreover, the spatial resolution is too poor to derive the fractionation in the different regions of the sources (warm inner envelope, cold outer envelope, outflows, etc.). Interferometry is the only possibility for going further in this study. In this respect, ALMA will allow considerable progress to be made.

Acknowledgements. B. Parise wishes to thank the IRAM $30 \mathrm{~m}$ operator Manuel Ruiz for his precious help during the observations.

\section{Appendix: Computation of error bars for the fractionation ratios}

The ratio of two Gaussian distributed variables $X$ and $Y$ is Gaussian only when $\sigma_{X} \ll \bar{X}$ and $\sigma_{Y} \ll \bar{Y}$. When the errors are too large, deviations from Gaussianity are observed and it is thus necessary to accurately compute the repartition function of the random variable $R=X / Y$. This repartition function is given by: $f_{R}(u)=\int_{-\infty}^{\infty} f_{X}(u y) f_{Y}(y)|y| \mathrm{d} y$.
This distribution is generally asymmetric, so it leads to asymmetric confidence intervals. We chose to give intervals associated to a $90 \%$ confidence level in this paper. As an example, we present in Fig. 6 the comparison with the repartition function for the $\mathrm{CH}_{2} \mathrm{DOH} / \mathrm{CH}_{3} \mathrm{OH}$ ratio in IRAS2, assuming that $\mathrm{CH}_{2} \mathrm{DOH}$ and $\mathrm{CH}_{3} \mathrm{OH}$ have a Gaussian distribution.

\section{References}

André, P., Ward-Thompson, D., \& Barsony, M. 2000, Protostars and Planets IV, 59

Bacmann, A., Lefloch, B., Ceccarelli, C., et al. 2002, A\&A, 389, L6 Bacmann, A., Lefloch, B., Ceccarelli, C., et al. 2003, ApJ, 585, L55

Bottinelli, S., Ceccarelli, C., Lefloch, B., et al. 2004, ApJ, 615, 354

Caselli, P., van der Tak, F. F. S., Ceccarelli, C., \& Bacmann, A. 2003, A\&A, 403, L37

Cazaux, S., Tielens, A. G. G. M., Ceccarelli, C., et al. 2003, ApJ, 593, L51

Ceccarelli, C., Castets, A., Loinard, L., Caux, E., \& Tielens, A. G. G. M. 1998, A\&A, 338, L43

Ceccarelli, C., Loinard, L., Castets, A., et al. 2001, A\&A, 372, 998

Cernis, K. 1990, Ap\&SS, 166, 315

Charnley, S. B., Tielens, A. G. G. M., \& Rodgers, S. D. 1997, ApJ, 482, L203

Dartois, E., Schutte, W., Geballe, T. R., et al. 1999, A\&A, 342, L32

Dartois, E., Thi, W.-F., Geballe, T. R., et al. 2003, A\&A, 399, 1009

Frerking, M. A., Langer, W. D., \& Wilson, R. W. 1982, ApJ, 262, 590

Geppert, W., et al. 2006, in Astrochemistry, Recent successes and current challenges, ed. D. C. Lis, in press

Herbst, E. 2005, in The Dusty and Molecular Universe: A Prelude to Herschel and ALMA, 205-210

Jørgensen, J. K., Schöier, F. L., \& van Dishoeck, E. F. 2002, A\&A, 389, 908 Jørgensen, J. K., Schöier, F. L., \& van Dishoeck, E. F. 2005, A\&A, 437, 501 Loinard, L., Castets, A., Ceccarelli, C., et al. 2000, A\&A, 359, 1169

Loinard, L., Castets, A., Ceccarelli, C., Caux, E., \& Tielens, A. G. G. M. 2001, ApJ, 552, 163

Loinard, L., Castets, A., Ceccarelli, C., et al. 2002, Planet. Space Sci., 50, 1205 Maret, S., Ceccarelli, C., Caux, E., et al. 2004, A\&A, 416, 577

Maret, S., Ceccarelli, C., Tielens, A. G. G. M., et al. 2005, A\&A, 442, 527

Menten, K. M., Walmsley, C. M., Henkel, C., \& Wilson, T. L. 1988, A\&A, 198, 253

Nagaoka, A., Watanabe, N., \& Kouchi, A. 2005, ApJ, 624, L29

Osamura, Y., Roberts, H., \& Herbst, E. 2004, A\&A, 421, 1101

Osamura, Y., Roberts, H., \& Herbst, E. 2005, ApJ, 621, 348

Parise, B., Ceccarelli, C., Tielens, A. G. G. M., et al. 2002, A\&A, 393, L49

Parise, B., Simon, T., Caux, E., et al. 2003, A\&A, 410, 897

Parise, B., Castets, A., Herbst, E., et al. 2004, A\&A, 416, 159

Parise, B., Caux, E., Castets, A., et al. 2005, A\&A, 431, 547

Roberts, H., \& Millar, T. J. 2000, A\&A, 364, 780

Roberts, H., Fuller, G. A., Millar, T. J., Hatchell, J., \& Buckle, J. V. 2002, A\&A, 381,1026

Roberts, H., Herbst, E., \& Millar, T. J. 2003, ApJ, 591, L41

Roberts, H., Herbst, E., \& Millar, T. J. 2004, A\&A, 424, 905

Stantcheva, T., \& Herbst, E. 2003, MNRAS, 340, 983

Tafalla, M., Myers, P. C., Caselli, P., Walmsley, C. M., \& Comito, C. 2002, ApJ, 569,815

Tielens, A. G. G. M. 1983, A\&A, 119, 177

Vastel, C., Phillips, T. G., \& Yoshida, H. 2004, ApJ, 606, L127 
B. Parise et al.: Deuterated formaldehyde and methanol in low-mass protostars, Online Material p 1

\section{Online Material}


B. Parise et al.: Deuterated formaldehyde and methanol in low-mass protostars, Online Material p 2
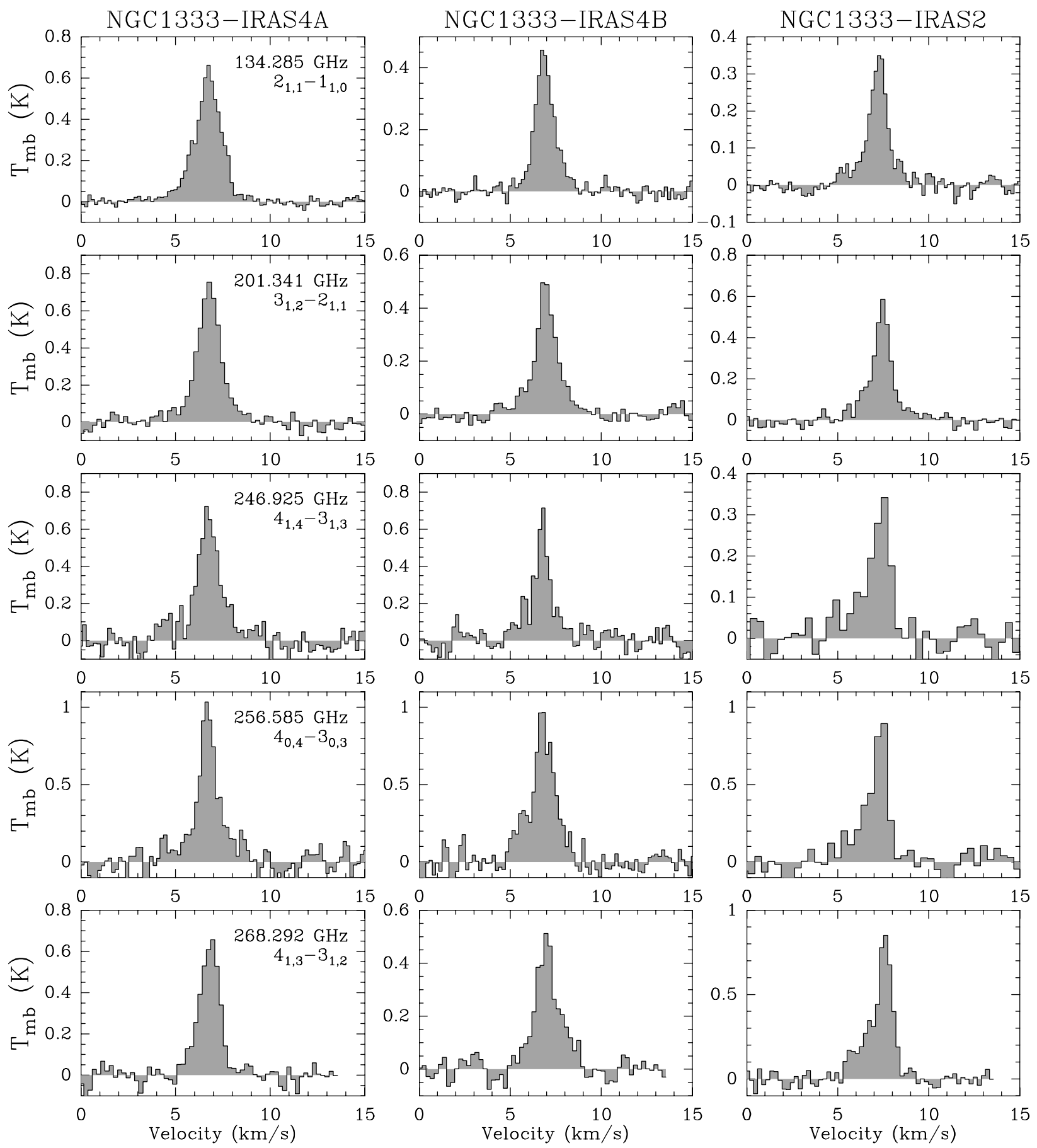

Fig. 7. HDCO lines for IRAS4a, IRAS4b, and IRAS2. 
B. Parise et al.: Deuterated formaldehyde and methanol in low-mass protostars, Online Material p 3
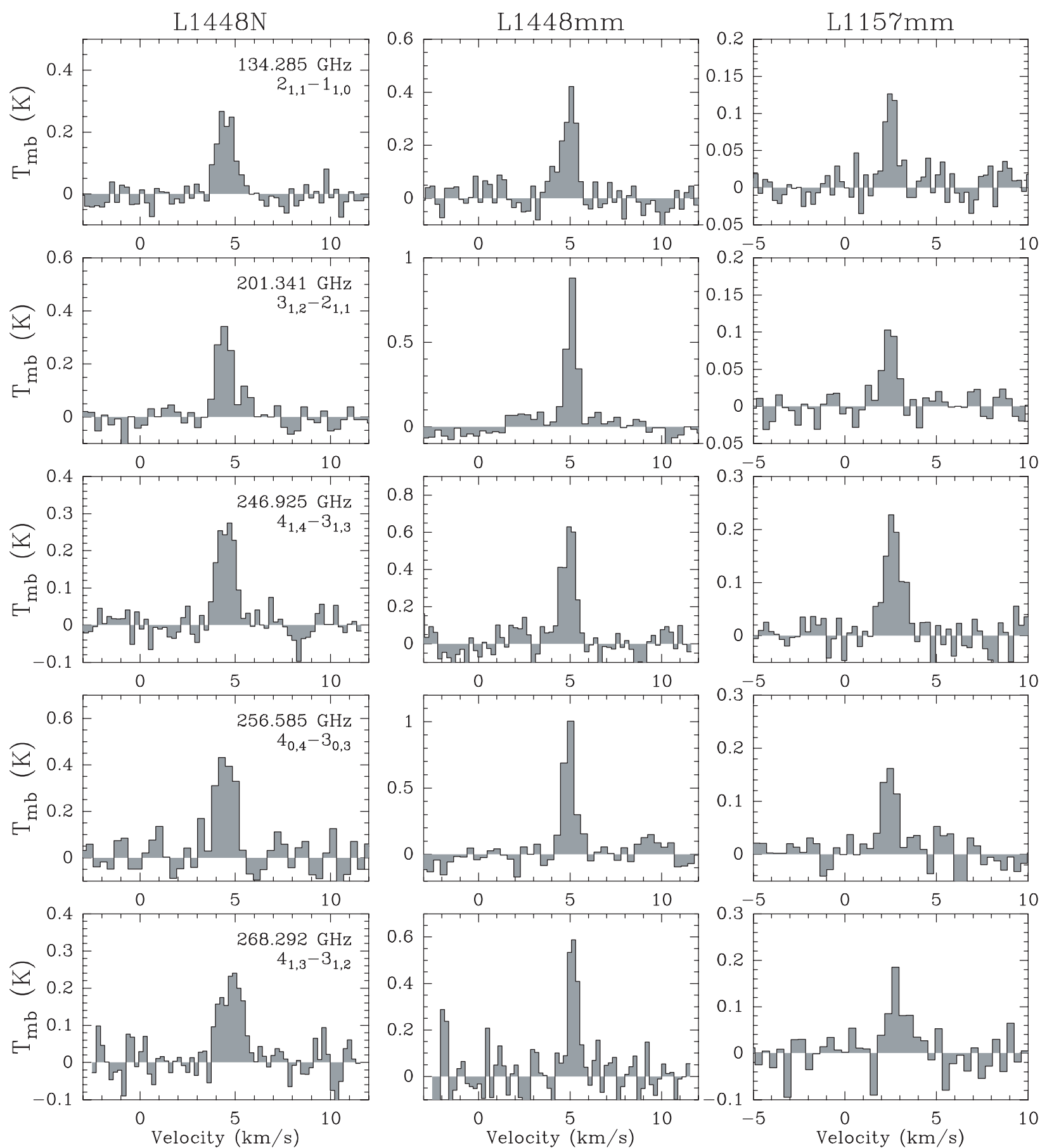

Fig. 8. HDCO lines for L1448N, L1448mm, and L1157mm. 
B. Parise et al.: Deuterated formaldehyde and methanol in low-mass protostars, Online Material p 4
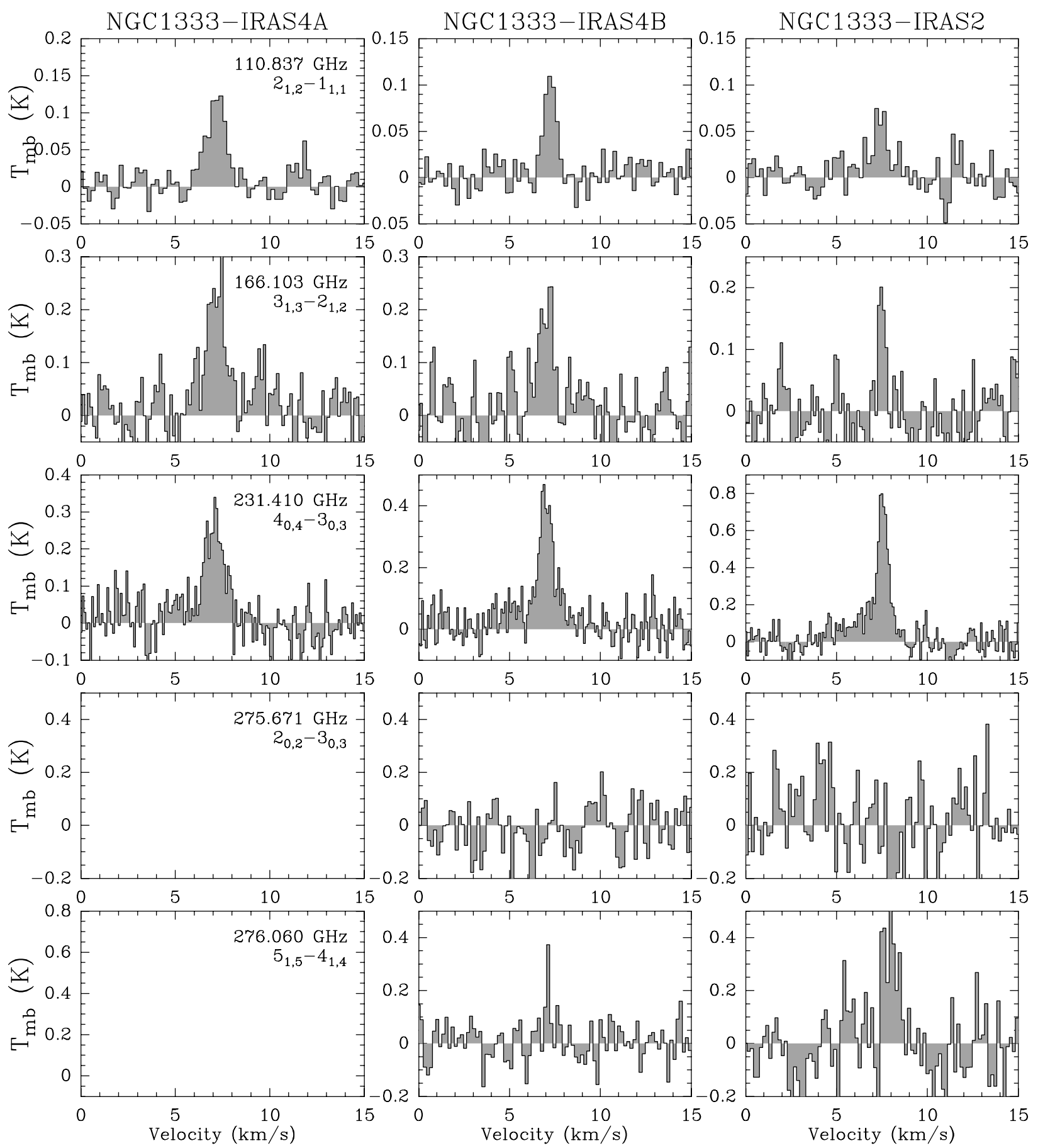

Fig. 9. $\mathrm{D}_{2} \mathrm{CO}$ lines for IRAS4a, IRAS4b, and IRAS2. 
B. Parise et al.: Deuterated formaldehyde and methanol in low-mass protostars, Online Material p 5
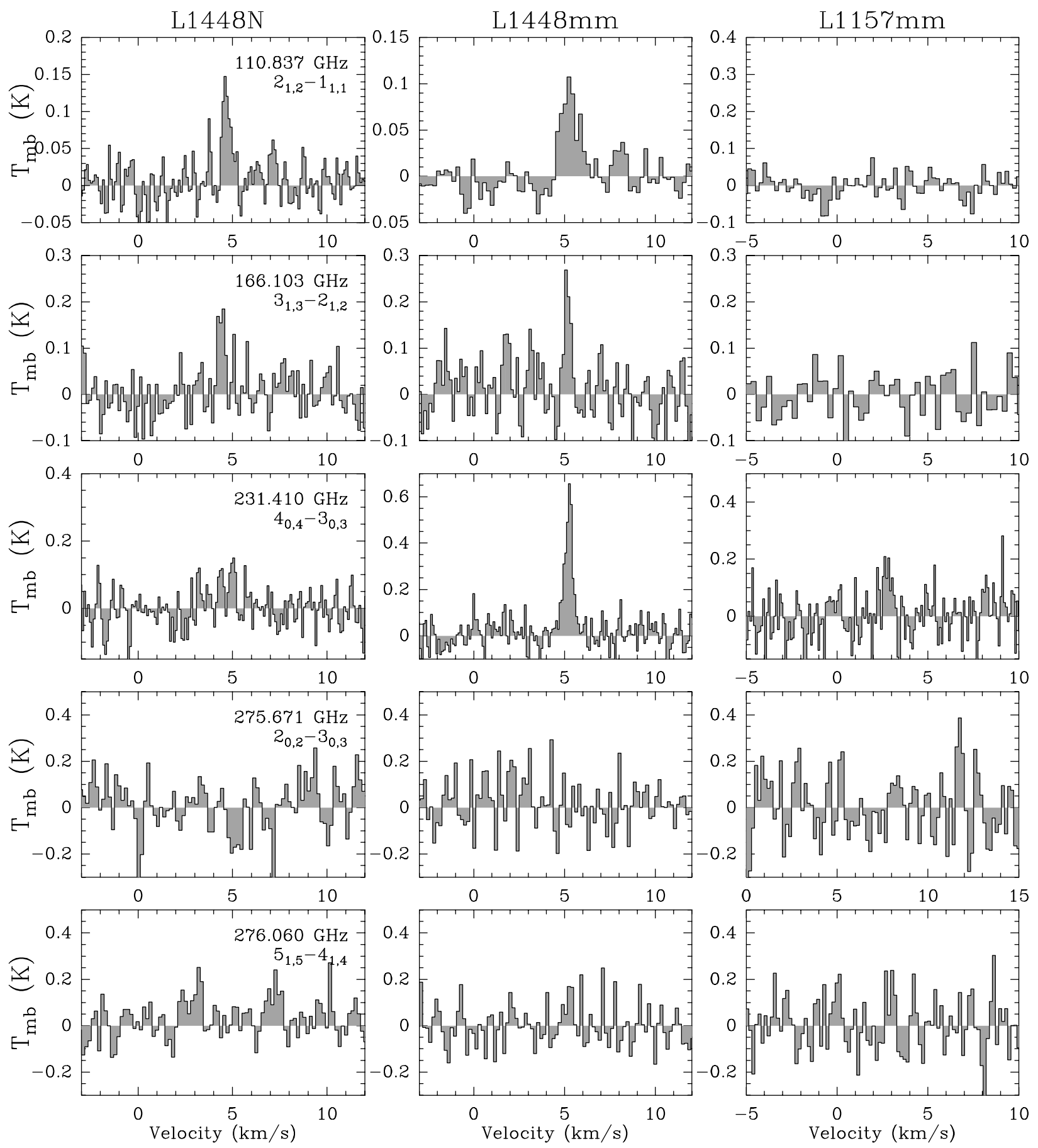

Fig. 10. $D_{2}$ CO lines for L1448N, L1448mm, and L1157mm. 
B. Parise et al.: Deuterated formaldehyde and methanol in low-mass protostars, Online Material p 6

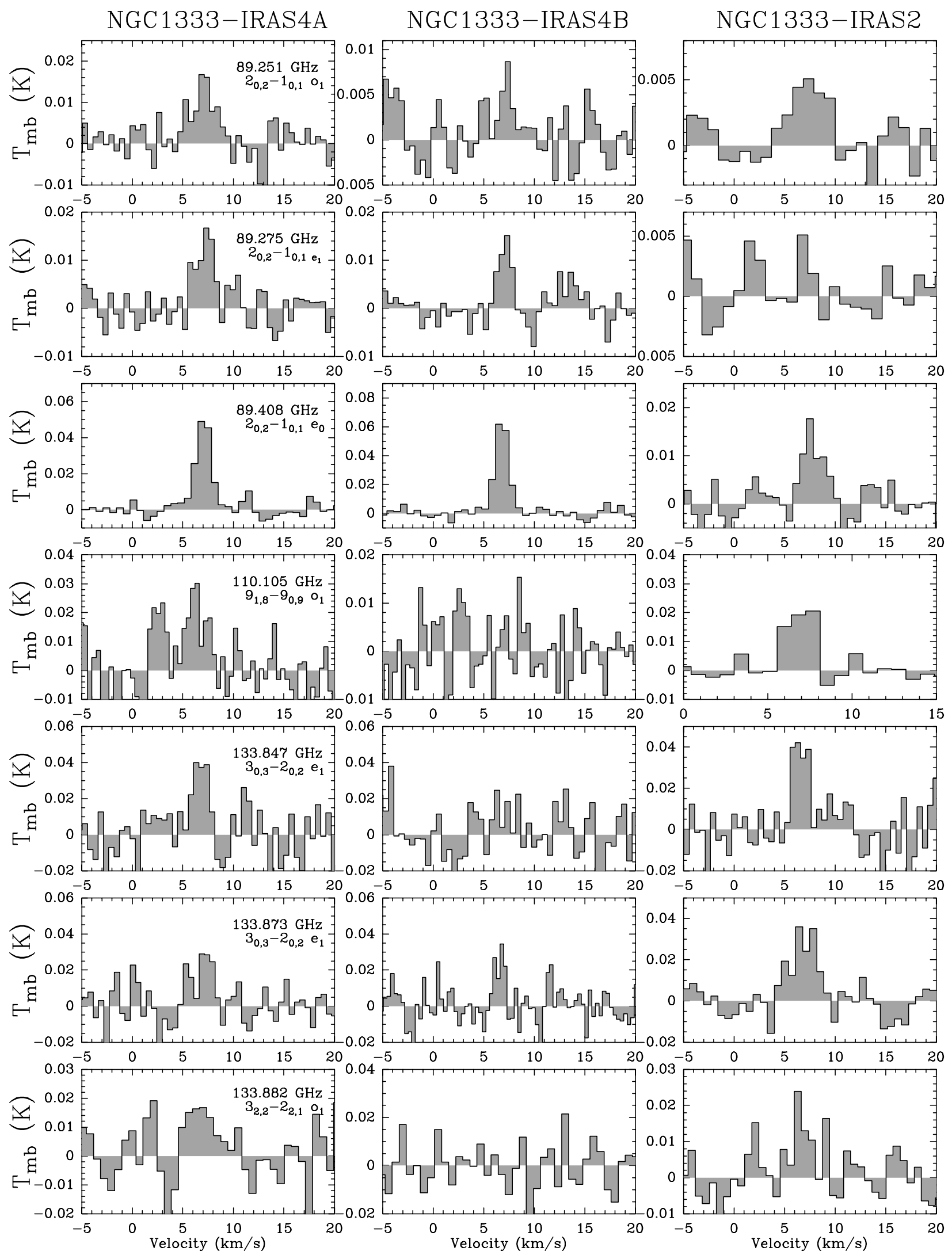

Fig. 11. $\mathrm{CH}_{2} \mathrm{DOH}$ lines for IRAS4a, IRAS4b, and IRAS2. 
B. Parise et al.: Deuterated formaldehyde and methanol in low-mass protostars, Online Material p 7
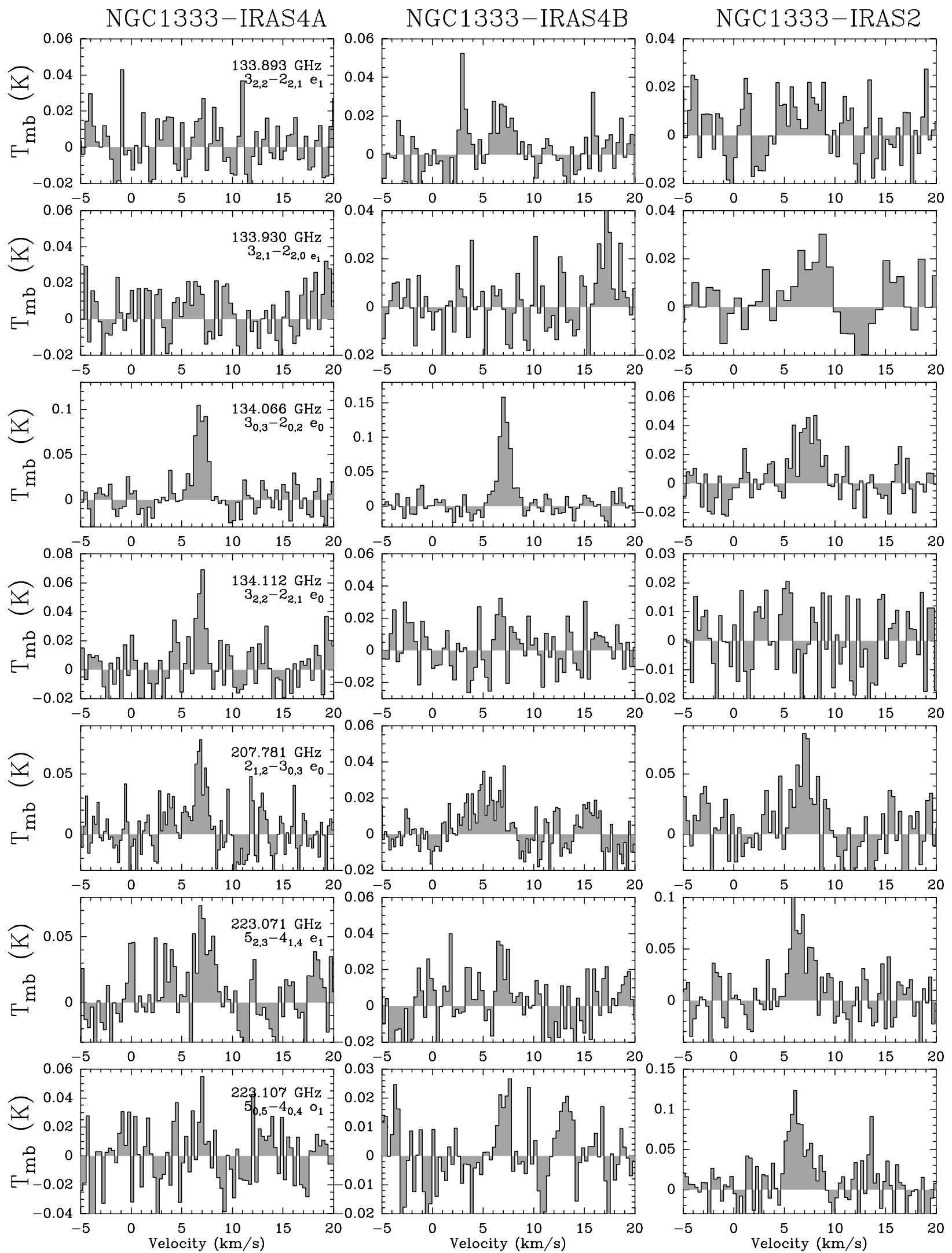

Fig. 12. $\mathrm{CH}_{2} \mathrm{DOH}$ lines for IRAS4a, IRAS4b, and IRAS2. 
B. Parise et al.: Deuterated formaldehyde and methanol in low-mass protostars, Online Material $p 8$

Table 6. Main-beam intensities, peak temperatures, and width for the observed transitions towards NGC1333-IRAS4A, IRAS4B, and IRAS2. The errors on the fluxes were computed as the quadratic sum of the statistical error and the calibration uncertainty (see text). Upper limits are $3 \sigma$ (see text).

\begin{tabular}{|c|c|c|c|c|c|c|c|c|c|c|}
\hline \multirow[b]{2}{*}{$\begin{array}{l}\text { Freq. } \\
\text { GHz }\end{array}$} & \multirow[b]{2}{*}{$\begin{array}{c}E_{\mathrm{up}} \\
\mathrm{cm}^{-1}\end{array}$} & \multicolumn{3}{|c|}{ IRAS4A } & \multicolumn{3}{|c|}{ IRAS4B } & \multicolumn{3}{|c|}{ IRAS2 } \\
\hline & & $\int_{\mathrm{K} \mathrm{km} \mathrm{s}^{-1}} T_{\mathrm{mb}} \mathrm{d} v$ & $\begin{array}{c}T_{\mathrm{mb}} \\
\mathrm{K}\end{array}$ & $\begin{array}{c}\Delta v \\
\mathrm{~km} \mathrm{~s}^{-1}\end{array}$ & $\int_{\mathrm{K} \mathrm{km} \mathrm{s}^{-1}} T_{\mathrm{mb}} \mathrm{d} v$ & $\begin{array}{c}T_{\mathrm{mb}} \\
\mathrm{K}\end{array}$ & $\begin{array}{c}\Delta v \\
\mathrm{~km} \mathrm{~s}^{-1}\end{array}$ & $\int_{\mathrm{K} \mathrm{km} \mathrm{s}^{-1}} T_{\mathrm{mb}} \mathrm{d} v$ & $\begin{array}{c}T_{\mathrm{mb}} \\
\mathrm{K}\end{array}$ & $\begin{array}{c}\Delta v \\
\mathrm{~km} \mathrm{~s}^{-1}\end{array}$ \\
\hline \multicolumn{11}{|l|}{$\mathrm{HDCO}$} \\
\hline 134.285 & 12.3 & $0.95 \pm 0.10$ & 0.58 & $1.5 \pm 0.1$ & $0.59 \pm 0.06$ & 0.43 & $1.3 \pm 0.1$ & $0.43 \pm 0.04$ & 0.33 & $1.2 \pm 0.1$ \\
\hline 201.341 & 19.0 & $1.09 \pm 0.11$ & .73 & $1.5 \pm 0.1$ & $.70 \pm 0.07$ & 0.47 & $1.4 \pm 0.1$ & $0.69 \pm 0.07$ & .53 & $1.2 \pm 0.1$ \\
\hline 246.924 & 26.1 & $0.98 \pm 0.11$ & 0.65 & $1.6 \pm 0.1$ & $0.74 \pm 0.10$ & 0.57 & $1.2 \pm 0.1$ & $0.50 \pm 0.08$ & 0.33 & $1.4 \pm 0.2$ \\
\hline 256.585 & 21.4 & $1.19 \pm 0.13$ & 0.94 & $1.2 \pm 0.1$ & $1.38 \pm 0.15$ & 0.83 & $1.6 \pm 0.1$ & $1.11 \pm 0.14$ & 0.92 & $1.1 \pm 0.1$ \\
\hline 268.292 & 27.9 & $0.82 \pm 0.13$ & 0.64 & $1.2 \pm 0.1$ & $0.74 \pm 0.12$ & 0.44 & $1.6 \pm 0.1$ & $1.00 \pm 0.16$ & 0.76 & $1.1 \pm 0.1$ \\
\hline \multicolumn{11}{|l|}{$\mathrm{D}_{2} \mathrm{CO}$} \\
\hline 110.838 & 9.3 & $0.16 \pm 0.01$ & 0.12 & $1.2 \pm 0.1$ & $0.095 \pm 0.011$ & 0.12 & $0.8 \pm 0.1$ & $0.077 \pm 0.017$ & 0.062 & $1.2 \pm 0.4$ \\
\hline 166.103 & 14.8 & $0.33 \pm 0.04$ & 0.24 & $1.3 \pm 0.2$ & $0.24 \pm 0.04$ & 0.22 & $1.0 \pm 0.2$ & $0.095 \pm 0.022$ & 0.21 & $0.4 \pm 0.1$ \\
\hline 231.410 & 19.4 & $0.37 \pm 0.05$ & 0.28 & $1.3 \pm 0.1$ & $0.47 \pm 0.06$ & 0.41 & $1.1 \pm 0.1$ & $0.63 \pm 0.07$ & 0.76 & $0.8 \pm 0.1$ \\
\hline 275.671 & 20.9 & - & - & - & $\leq 0.16$ & - & - & $\leq 0.26$ & - & - \\
\hline 276.060 & 31.4 & - & - & - & $0.10 \pm 0.03$ & 0.46 & $0.2 \pm 0.1$ & $0.44 \pm 0.10$ & 0.45 & $0.9 \pm 0.2$ \\
\hline \multicolumn{11}{|c|}{$\mathrm{CH}_{2} \mathrm{DOH}$} \\
\hline 89.251 & 17.2 & $0.042 \pm 0.008$ & 0.014 & $2.8 \pm 0.6$ & $0.011 \pm 0.005$ & 0.008 & $1.3 \pm 0.8$ & $0.021 \pm 0.008$ & 0.006 & $3.4 \pm 1.2$ \\
\hline 89.275 & 13.8 & $0.033 \pm 0.007$ & 0.015 & $2.0 \pm 0.5$ & $0.023 \pm 0.004$ & 0.015 & $1.4 \pm 0.3$ & $\leq 0.013$ & - & - \\
\hline 89.408 & 4.5 & $0.098 \pm 0.008$ & 0.054 & $1.7 \pm 0.1$ & $0.113 \pm 0.008$ & 0.072 & $1.5 \pm 0.1$ & $0.038 \pm 0.005$ & 0.015 & $2.3 \pm 0.4$ \\
\hline 110.105 & 83.4 & $0.054 \pm 0.014$ & 0.022 & $2.3 \pm 0.6$ & $\leq 0.027$ & - & - & $0.050 \pm 0.009$ & 0.025 & $1.8 \pm 3.4$ \\
\hline 133.847 & 18.3 & $0.084 \pm 0.022$ & 0.046 & $1.7 \pm 0.4$ & $\leq 0.077$ & - & - & $0.090 \pm 0.017$ & 0.045 & $1.9 \pm 0.3$ \\
\hline 133.873 & 21.7 & $0.064 \pm 0.019$ & 0.025 & $2.4 \pm 0.7$ & $0.035 \pm 0.009$ & 0.030 & $1.1 \pm 0.2$ & $0.104 \pm 0.020$ & 0.034 & $2.9 \pm 0.5$ \\
\hline 133.882 & 33.6 & $0.054 \pm 0.019$ & 0.018 & $2.6 \pm 0.9$ & $\leq 0.039$ & - & - & $0.048 \pm 0.026$ & 0.016 & $2.9 \pm 2.0$ \\
\hline 133.893 & 27.4 & $\leq 0.052$ & - & - & $0.055 \pm 0.018$ & 0.024 & $2.1 \pm 0.7$ & $\leq 0.10$ & - & - \\
\hline 133.930 & 27.4 & $\leq 0.052$ & - & - & $\leq 0.039$ & - & - & $0.080 \pm 0.022$ & 0.025 & $3.0 \pm 0.7$ \\
\hline 134.066 & 8.9 & $157 \pm 0.022$ & 0.107 & $1.4 \pm 0.2$ & $0.197 \pm 0.024$ & 0.160 & $1.2 \pm 0.1$ & $0.115 \pm 0.021$ & 0.042 & $2.6 \pm 0.4$ \\
\hline 134.112 & 20.2 & $0.068 \pm 0.015$ & 0.071 & $0.9 \pm 0.2$ & $\leq 0.052$ & - & - & $\leq 0.065$ & - & - \\
\hline 207.781 & 15.9 & $0.094 \pm 0.017$ & 0.067 & $1.3 \pm 0.2$ & $0.089 \pm 0.017$ & 0.024 & $3.6 \pm 0.6$ & $0.144 \pm 0.029$ & 0.067 & $2.0 \pm 0.5$ \\
\hline 223.071 & 33.6 & $0.116 \pm 0.022$ & 0.059 & $1.9 \pm 0.3$ & $0.042 \pm 0.011$ & 0.034 & $1.1 \pm 0.3$ & $0.229 \pm 0.035$ & 0.081 & $2.7 \pm 0.4$ \\
\hline 223.107 & 35.1 & $\leq 0.10$ & - & - & $0.030 \pm 0.010$ & 0.024 & $1.2 \pm 0.4$ & $0.234 \pm 0.041$ & 0.098 & $2.3 \pm 0.4$ \\
\hline 223.128 & 40.8 & $\leq 0.083$ & - & - & $0.046 \pm 0.013$ & 0.037 & $1.2 \pm 0.4$ & $0.189 \pm 0.055$ & 0.073 & $2.4 \pm 0.8$ \\
\hline 223.131 & 79.4 & $0.015 \pm 0.01$ & 0.025 & $0.6 \pm 0.2$ & $0.02 \pm 0.01$ & 0.02 & $0.9 \pm 0.3$ & $0.04 \pm 0.03$ & 0.016 & $2.3 \pm 1.2$ \\
\hline 223.131 & 79.4 & $0.015 \pm 0.01$ & 0.025 & $0.6 \pm 0.2$ & $0.02 \pm 0.01$ & 0.02 & $0.9 \pm 0.3$ & $0.04 \pm 0.03$ & 0.016 & $2.3 \pm 1.2$ \\
\hline 223.154 & 60.8 & $0.046 \pm 0.02$ & 0.02 & $2.0 \pm 0.5$ & $0.02 \pm 0.01$ & 0.015 & $1.2 \pm 0.5$ & $0.13 \pm 0.03$ & 0.05 & $2.6 \pm 0.4$ \\
\hline 223.154 & 60.8 & $0.046 \pm 0.02$ & 0.02 & $2.0 \pm 0.5$ & $0.02 \pm 0.01$ & 0.015 & $1.2 \pm 0.5$ & $0.13 \pm 0.03$ & 0.05 & $2.6 \pm 0.4$ \\
\hline 223.315 & 40.8 & $0.061 \pm 0.016$ & 0.040 & $1.4 \pm 0.4$ & $0.083 \pm 0.015$ & 0.032 & $2.5 \pm 0.4$ & $0.192 \pm 0.032$ & 0.061 & $3.0 \pm 0.4$ \\
\hline 223.422 & 33.6 & $0.126 \pm 0.024$ & 0.061 & $2.0 \pm 0.4$ & $0.062 \pm 0.010$ & 0.042 & $1.4 \pm 0.2$ & $0.229 \pm 0.038$ & 0.064 & $3.4 \pm 0.5$ \\
\hline \multicolumn{11}{|l|}{$\mathrm{CH}_{3} \mathrm{OD}$} \\
\hline 110189 & & $\leq 0.0$ & - & - & $0.022 \pm$ & 0.054 & $0.5 \pm 0.2$ & $\leq 0$. & - & - \\
\hline 110.263 & 10.8 & $0.041 \pm 0.014$ & 0.018 & $2.0 \pm 0.7$ & $\leq 0.03$ & - & - & $\leq 0.02$ & - & - \\
\hline 110.476 & 15.4 & $\leq 0.02$ & - & - & $\leq 0.02$ & - & - & $\leq 0.02$ & - & - \\
\hline 133.925 & 6.0 & $0.076 \pm 0.020$ & 0.024 & $2.7 \pm 0.8$ & $0.046 \pm 0.015$ & 0.038 & $1.1 \pm 0.5$ & $\leq 0.05$ & - & - \\
\hline 223.309 & 26.8 & $\leq 0.07$ & - & - & $0.035 \pm 0.009$ & 0.029 & $1.2 \pm 0.3$ & $\leq 0.15$ & - & - \\
\hline 226.539 & 22.7 & $\leq 0.06$ & - & - & $\leq 0.04$ & - & - & $\leq 0.17$ & - & - \\
\hline \multicolumn{11}{|c|}{$\mathrm{CHD}_{2} \mathrm{OH}$} \\
\hline 83.1292 & 17.0 & $\leq 0.03$ & - & - & 005 & 0.026 & $0.7 \pm$ & $\leq 0$. & - & - \\
\hline 83.2895 & 4.2 & $0.009 \pm 0.002$ & 0.016 & $0.5 \pm 0.1$ & $0.024 \pm 0.004$ & 0.027 & $0.9 \pm 0.2$ & $\leq 0.021$ & - & - \\
\hline 83.3036 & 10.3 & $0.015 \pm 0.006$ & - & - & $0.011 \pm 0.004$ & 0.012 & $0.9 \pm 0.3$ & $\leq 0.021$ & - & - \\
\hline 207.771 & 33.6 & $0.066 \pm 0.040$ & 0.022 & $2.8 \pm 1.7$ & $0.089 \pm 0.025$ & 0.025 & $3.4 \pm 0.9$ & $0.115 \pm 0.041$ & 0.029 & $3.7 \pm 1.4$ \\
\hline 207.827 & 42.6 & $\leq 0.07$ & - & - & $0.097 \pm 0.027$ & 0.021 & $4.4 \pm 1.0$ & $0.087 \pm 0.045$ & 0.016 & $5.2 \pm 2.3$ \\
\hline 207.864 & 68.4 & $0.062 \pm 0.030$ & 0.036 & $1.6 \pm 0.7$ & $\leq 0.035$ & - & - & $0.088 \pm 0.033$ & 0.035 & $2.3 \pm 1.0$ \\
\hline 207.868 & 53.5 & $0.12 \pm 0.04$ & 0.03 & $3.7 \pm 0.8$ & $0.034 \pm 0.011$ & 0.02 & $1.8 \pm 0.4$ & $0.13 \pm 0.03$ & 0.03 & $4.8 \pm 0.9$ \\
\hline 207.869 & 53.5 & $0.12 \pm 0.04$ & 0.03 & $3.7 \pm 0.8$ & $0.034 \pm 0.011$ & 0.02 & $1.8 \pm 0.4$ & $0.13 \pm 0.03$ & 0.03 & $4.8 \pm 0.9$ \\
\hline
\end{tabular}


B. Parise et al.: Deuterated formaldehyde and methanol in low-mass protostars, Online Material p 9

Table 7. Main-beam intensities, peak temperatures, and width for the detected transitions towards L1448N, L1448mm, and L1157mm. The errors on the fluxes were computed as the quadratic sum of the statistical error and the calibration uncertainty (see text). Upper limits are $3 \sigma$. Upper limits for lines not detected in any of these three sources can be found in Table 9.

\begin{tabular}{|c|c|c|c|c|c|c|c|c|c|c|}
\hline \multirow[b]{2}{*}{$\begin{array}{l}\text { Freq. } \\
\text { GHz }\end{array}$} & \multirow[b]{2}{*}{$\begin{array}{c}E_{\mathrm{up}} \\
\mathrm{cm}^{-1}\end{array}$} & \multicolumn{3}{|c|}{ L1448N } & \multicolumn{3}{|c|}{$\mathrm{L} 1448 \mathrm{~mm}$} & \multicolumn{3}{|c|}{$\mathrm{L} 1157 \mathrm{~mm}$} \\
\hline & & $\int_{\mathrm{K} \mathrm{km} \mathrm{s}^{-1}} T_{\mathrm{mb}} \mathrm{d} v$ & $\begin{array}{c}T_{\mathrm{mb}} \\
\mathrm{K}\end{array}$ & $\begin{array}{c}\Delta v \\
\mathrm{~km} \mathrm{~s}^{-1}\end{array}$ & $\int_{\mathrm{K} \mathrm{km} \mathrm{s}^{-1}} T_{\mathrm{mb}} \mathrm{d} v$ & $\begin{array}{c}T_{\mathrm{mb}} \\
\mathrm{K}\end{array}$ & $\begin{array}{c}\Delta v \\
\mathrm{~km} \mathrm{~s}^{-1}\end{array}$ & $\int_{\mathrm{K} \mathrm{km} \mathrm{s}^{-1}} T_{\mathrm{mb}} \mathrm{d} v$ & $\begin{array}{c}T_{\mathrm{mb}} \\
\mathrm{K}\end{array}$ & $\begin{array}{c}\Delta v \\
\mathrm{~km} \mathrm{~s}^{-1}\end{array}$ \\
\hline \multicolumn{11}{|l|}{ HDCO } \\
\hline 134.285 & 12.3 & $0.32 \pm 0.04$ & 0.26 & $1.1 \pm 0.1$ & $0.38 \pm 0.06$ & 0.38 & $0.9 \pm 0.1$ & $0.11 \pm 0.01$ & 0.13 & $0.8 \pm 0.1$ \\
\hline 201.341 & 19.0 & $0.36 \pm 0.05$ & 0.38 & $1.0 \pm 0.1$ & $0.53 \pm 0.06$ & 0.97 & $0.6 \pm 0.1$ & $0.10 \pm 0.03$ & 0.12 & $0.8 \pm 0.1$ \\
\hline 246.924 & 26.1 & $0.35 \pm 0.04$ & 0.30 & $1.1 \pm 0.1$ & $0.61 \pm 0.08$ & 0.63 & $0.9 \pm 0.1$ & $0.26 \pm 0.03$ & 0.20 & $1.2 \pm 0.1$ \\
\hline 256.585 & 21.4 & $0.60 \pm 0.10$ & 0.47 & $1.2 \pm 0.2$ & $0.79 \pm 0.10$ & 1.11 & $0.7 \pm 0.1$ & $0.18 \pm 0.03$ & 0.18 & $0.9 \pm 0.1$ \\
\hline 268.292 & 27.9 & $0.34 \pm 0.08$ & 0.24 & $1.4 \pm 0.3$ & $0.40 \pm 0.08$ & 0.66 & $0.6 \pm 0.1$ & $0.15 \pm 0.05$ & 0.20 & $0.7 \pm 0.4$ \\
\hline \multicolumn{11}{|l|}{$\mathrm{D}_{2} \mathrm{CO}$} \\
\hline 110.838 & 9.3 & $0.074 \pm 0.010$ & 0.137 & $0.5 \pm 0.1$ & $0.113 \pm 0.014$ & 0.10 & $1.1 \pm 0.1$ & $\leq 0.67$ & - & - \\
\hline 166.103 & 14.8 & $0.088 \pm 0.018$ & 0.204 & $0.4 \pm 0.1$ & $0.114 \pm 0.021$ & 0.27 & $0.4 \pm 0.1$ & $\leq 0.10$ & - & - \\
\hline 231.410 & 19.4 & $\leq 0.11$ & - & - & $0.325 \pm 0.038$ & 0.59 & $0.5 \pm 0.4$ & $0.109 \pm 0.031$ & 0.182 & $0.6 \pm 0.2$ \\
\hline 275.671 & 20.9 & $\leq 0.20$ & - & - & $\leq 0.16$ & - & - & $\leq 0.26$ & - & - \\
\hline 276.060 & 31.4 & $\leq 0.13$ & - & - & $0.092 \pm 0.051$ & 0.14 & $0.6 \pm 0.4$ & $\leq 0.21$ & - & - \\
\hline \multicolumn{11}{|c|}{$\mathrm{CH}_{2} \mathrm{DOH}$} \\
\hline 89.408 & & $0.029 \pm 0.002$ & 0.053 & $0.5 \pm 0.1$ & $0.040 \pm 0.007$ & 0.034 & $1.1 \pm 0.2$ & $0.021 \pm 0.004$ & 0.057 & $0.3 \pm 1.0$ \\
\hline \multicolumn{11}{|l|}{$\mathrm{CH}_{3} \mathrm{OD}$} \\
\hline 110.189 & & $\leq 0.04$ & - & - & $0.078 \pm 0.010$ & 0.073 & $1.0 \pm 0.1$ & $0.025 \pm 0.013$ & 0.050 & $0.5 \pm 0.3$ \\
\hline
\end{tabular}


B. Parise et al.: Deuterated formaldehyde and methanol in low-mass protostars, Online Material p 10

Table 8. Main-beam intensities, peak temperatures and width for the observed transitions towards L1527.

\begin{tabular}{lcccc}
\hline \hline & & \multicolumn{3}{c}{$\mathrm{L} 1527$} \\
$\begin{array}{l}\text { Frequency } \\
\mathrm{GHz}\end{array}$ & $\begin{array}{c}E_{\mathrm{up}} \\
\mathrm{cm}^{-1}\end{array}$ & $\begin{array}{c}\mathrm{K} \mathrm{km} \mathrm{s}_{\mathrm{mb}} \mathrm{d} v \\
T_{\mathrm{mb}} \\
\mathrm{K}\end{array}$ & $\begin{array}{c}\Delta v \\
\mathrm{~km} \mathrm{~s}^{-1}\end{array}$ \\
\hline $\mathrm{HDCO}$ & & & & \\
\hline 134.285 & 12.3 & $0.75 \pm 0.08$ & 1.1 & $0.7 \pm 0.1$ \\
201.341 & 19.0 & $0.70 \pm 0.08$ & 0.98 & $0.7 \pm 0.1$ \\
256.585 & 21.4 & $0.77 \pm 0.10$ & 0.18 & $0.9 \pm 0.1$ \\
\hline $\mathrm{D}_{2} \mathrm{CO}$ & & & & \\
\hline 110.838 & 9.3 & $0.133 \pm 0.016$ & 0.243 & $0.5 \pm 0.1$ \\
166.103 & 14.8 & $0.219 \pm 0.038$ & 0.327 & $0.6 \pm 0.1$ \\
231.410 & 19.4 & $0.367 \pm 0.047$ & 0.601 & $0.6 \pm 0.1$ \\
276.060 & 31.4 & $\leq 0.23$ & - & - \\
\hline
\end{tabular}

Table 9. Main-beam temperature upper limits at $3 \sigma$ level for the deuterated methanol transitions observed towards L1448N, L1448mm, and $\mathrm{L} 1157 \mathrm{~mm}$.

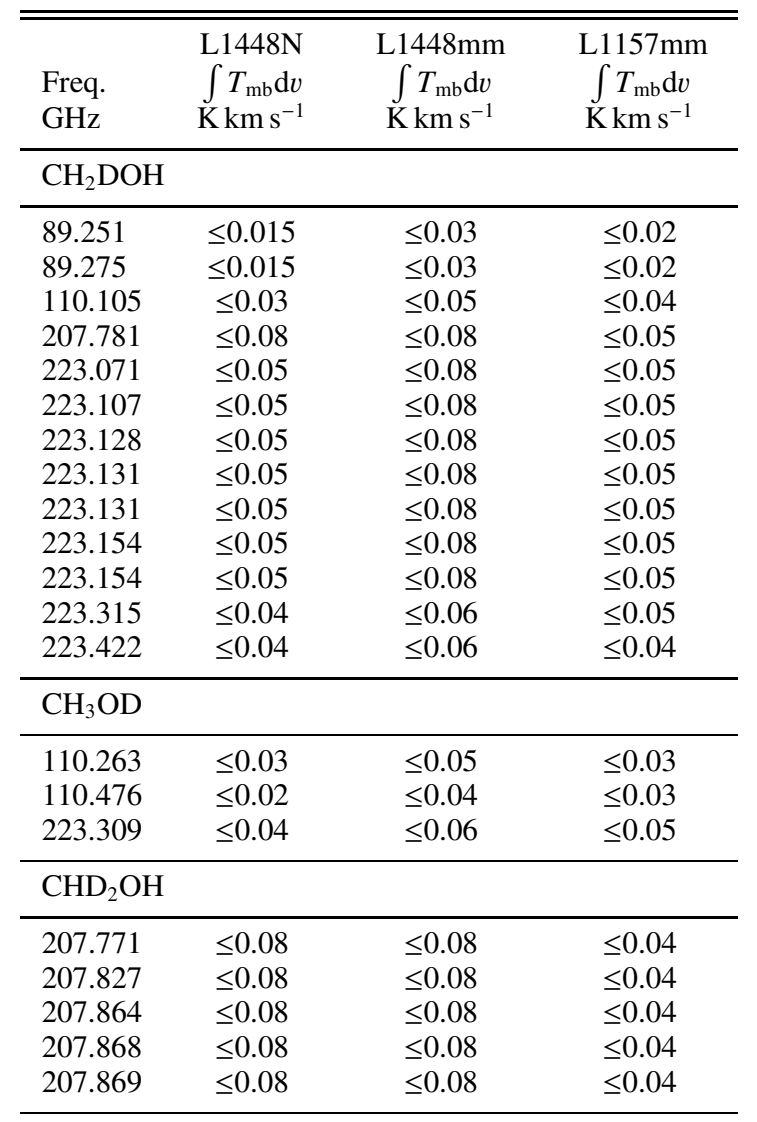

